\title{
RATIONAL MOORE $G$-SPACES
}

\author{
PETER J. KAHN
}

\begin{abstract}
This paper obtains some existence and uniqueness results for Moore spaces in the context of the equivariant homotopy theory of Bredon. This theory incorporates fixed-point-set data as part of the structure and so is a refinement of the classical equivariant homotopy theory. To avoid counterexamples to existence in the classical case and to focus on new phenomena involving the fixed-point-set structure, most of the results involve rational spaces. In this setting, there are no obstacles to existence, but a notion of projective dimension presents an obstacle to uniqueness: uniqueness is proved, subject to constraint on the projective dimension, and an example shows that this constraint is sharp. Various related existence results are proved and computations are given of certain equivariant mapping sets $[X, Y], X$ an equivariant Moore space.
\end{abstract}

1. Introduction. This paper presents some existence and uniqueness results for Moore spaces in the context of equivariant homotopy theory. In addition it also computes certain equivariant mapping sets $[X, Y]$, in which $X$ is an equivariant Moore space.

Throughout, $G$ will denote a finite group and all spaces, maps, homotopies, and actions will be pointed. By equivariant homotopy theory we mean the theory developed, for example, by Bredon [B] (or by Waner [Wn]). Thus, we consider not only $G$-spaces $X$, but we also systematically include information about all the $H$-fixed-point sets $X^{H}$ as $H$ ranges over the subgroups of $G$.

Following Bredon $[\mathbf{B}]$, a convenient way to do this uses the orbit category $O_{G}$, whose objects are the left coset spaces $G / H, H \leq G$, and whose morphisms are their $G$-maps. For example, the (reduced) homology $\underline{\tilde{H}}_{*} X$ of a $G$-space $X$ is taken to be a certain contravariant functor from $O_{G}$ to (graded) abelian groups. On objects $G / H$ of $O_{G}, \underline{\tilde{H}}_{*} X$ satisfies

$$
\underline{\tilde{H}}_{*} X(G / H)=\tilde{H}_{*}\left(X^{H}\right) .
$$

Homotopy groups $\underline{\pi}_{*} X$ are defined analogously (see 2.1).

A contravariant functor from $O_{G}$ to abelian groups is called an $O_{G}$-module. Let $\underline{M}$ be one such, and let $n \geq 2$ be an integer. A Moore $G$-space of type $(\underline{M}, n)$ is, then, a $G$-space $X$ such that

(1) $X^{H}$ is 1-connected, for all $H \leq G$,

(2) $\underline{\tilde{H}}_{n} X \approx \underline{M}$, as $O_{G}$-modules,

(3) $\underline{\tilde{H}}_{i} X=0, \quad i \neq n$.

Received by the editors April 22, 1985.

1980 Mathematics Subject Classification (1985 Revision). Primary 55P99, 57S17, 55P62, $55 \mathrm{Q} 05$.

Key words and phrases. Moore space, equivariant, $G$-space, homotopy.

The author was supported in part by NSF Grant DMS 8201029. 
We also say that $X$ realizes the $O_{G}$-module $\underline{M}$ (in degree $n$ ).

When $G=e$, the trivial group, the above definition reduces to the standard nonequivariant one. Moore $G$-spaces, as defined above, play a central role in the notion of equivariant homology decomposition [K2].

If $H$-fixed sets $X^{H}$ are disregarded in (1.1) when $H \neq e$, then we obtain what we call a "classical" Moore $G$-space. More precisely, let $M$ be a $\mathbf{Z} G$-module and $n \geq 2$. Then a classical Moore $G$-space of type $(M, n)$ is a $G$-space $X$ such that

(1) $X$ is 1-connected,

(2) $\tilde{H}_{n} X \approx M$, as $\mathbf{Z} G$-modules,

(3) $\tilde{H}_{i} X=0, \quad i \neq n$.

Now even in this simpler classical case, general questions of existence and uniqueness are quite difficult. There are a number of special existence theorems (e.g., $[\mathbf{A}, \mathbf{V}]$ ) and some nonexistence results (e.g., $[\mathbf{C 1}, \mathbf{K 1}]$ ), but no general picture has emerged.

In this paper, we wish to avoid all these classical obstacles and focus on the simplest phenomena that may lie beyond them when $H$-fixed sets are taken into account. Accordingly, we consider the following conditions.

(1) Abelian groups are vector spaces over the field $\mathbf{Q}$ of rational numbers.

(2) Spaces are 1-connected, rational $\mathrm{CW}$ complexes.

We shall invoke these restrictions (explicitly) in many of our results below. Note that we intend (2) to apply, in particular, to all the fixed sets of a $G$-complex.

Conditions (1.3) do suffice to kill the unwanted, classical obstacles (see Corollary E). Indeed, they kill all serious obstacles to existence of general Moore $G$-spaces of type $(\underline{M}, n)$. When each $\underline{M}(G / H)$ is finite-dimensional, $H \leq G$, this follows immediately from a result of Triantafillou [T2] on the existence of rational $G$-spaces with prescribed cohomology. When $\underline{M}$ is arbitrary and $n \geq$ projective dimension $\underline{M}$, the assertion is a specialization of Theorem 3.3. (We describe the notion of projective dimension of $O_{G}$-modules in 2.2.2.) In $\S 4$ we give some details, and, combining the above with a realizability result (Theorem 3.1) first discovered by W. Lück [L], we obtain a refinement (see Theorem 4.1), namely, assuming (1.3), every free $O_{G}$-resolution may be realized by a Moore $G$-complex.

The simplest nonclassical phenomenon that is not trivialized by conditions (1.3) involves the projective dimension of $O_{G}$-modules mentioned above. The reader is referred to [T1] for the development of a number of important properties of this invariant. For example, in contrast to the classical projective dimension of $\mathbf{Q}[G]$ modules, which is always zero, the projective dimension of $O_{G}$-modules may be large, even in the presence of conditions (1.3). Note, however, that for each $G$, there is a nonnegative integer $l(G) \leq \log _{2}|G|$ which is a bound on this projective dimension. This fact is first stated and proved in $[\mathbf{R} \& \mathbf{T}]$, but it is already implicit in the constructions of $[\mathbf{T 1}]$. We define $l(G)$ in 2.2.2.

This phenomenon presents the basic obstacle to our constructions. Theorem $B$ shows that the obstacle is essential. We get around it by requiring that the projective dimension be bounded by (approximately) the degree $n$ of the Moore $G$ space $X$. This condition arises from a simple stable range fact (Lemma 3.10) used in the constructions; it is always satisfied by sufficiently high suspensions $\sum^{l} X$. Hence, we shall refer to it as a stability condition. 
TheOREM A (COROllary 4.6). Assume (1.3). All Moore G-spaces realizing a fixed $O_{G}$-module $\underline{M}$ have the same stable $G$-homotopy-type. In fact, if

$$
n \geq \operatorname{proj} \operatorname{dim} \underline{M}+1,
$$

then all Moore $G$-spaces of type $(\underline{M}, n)$ are $G$-equivalent.

The stability condition $n \geq \operatorname{proj} \operatorname{dim} \underline{M}+1$ is needed for uniqueness, as the next result shows.

THEOREM B (THEOREM 5.1). Let $G=\mathbf{Z} / 2 \oplus \mathbf{Z} / 2$. There exists an $O_{G^{-}}$ module $\underline{M}$ of projective dimension two and two Moore $G$-spaces $L_{1}, L_{2}$ of type $(\underline{M}, 2)$ that are not $G$-equivalent. Of course, by Theorem $\mathrm{A}, \sum L_{1}$ is $G$-equivalent to $\sum L_{2}$.

For the next result, we need, for any $G$-spaces $X, Y$, the set $[X, Y]$ of $G$-homotopy classes of $G$-maps $X \rightarrow Y$, and the abelian group $\{X, Y\}=\operatorname{colim}\left[\sum^{l} X, \sum^{l} Y\right]$. We also need, for any $O_{G}$-modules $\underline{M}, \underline{N}$, the abelian group $\operatorname{Hom}(\underline{M}, \underline{N})$ of natural transformations $\underline{M} \rightarrow \underline{N}$, and $\operatorname{Ext}^{i}(\underline{M}, \underline{N})$, where $\operatorname{Ext}^{i}$ is the $i$ th derived functor of Hom. Let $\mathrm{Ext}^{i}=0$ for $i<0$.

THEOREM C. Assume (1.3). Suppose that $K_{i}$ is a Moore G-space of type $\left(\underline{M}_{i}, n_{i}\right), i=1,2$. Then there is a natural isomorphism of abelian groups

$$
\left\{K_{1}, K_{2}\right\} \approx \operatorname{Ext}^{n_{2}-n_{1}}\left(\underline{M}_{1}, \underline{M}_{2}\right) .
$$

When $n_{1}=n_{2}=n$, this is just the map induced by $\underline{\tilde{H}}_{n}$. The natural map $\left[K_{1}, K_{2}\right] \rightarrow\left\{K_{1}, K_{2}\right\}$ is

(1) surjective, if $2 n_{2}-n_{1} \geq \operatorname{proj} \operatorname{dim} \underline{M}_{1}+1$,

(2) bijective, if $2 n_{2}-n_{1} \geq \operatorname{proj} \operatorname{dim} \underline{M}_{1}+2$.

Theorem $\mathrm{A}$ is an easy consequence of Theorem $\mathrm{C}$ (see end of $\S 4$ ).

Notice that in the example of Theorem B the natural map $\left[L_{1}, L_{2}\right] \rightarrow\left\{L_{1}, L_{2}\right\}$ is not surjective and that the required inequality in (1) above indeed fails to hold by one.

As a "best-case" illustration, we next specialize to the case in which $\underline{M}$ has small projective dimension. We do not assume (1.3). The method of proof here is simpler than that used for the more general results above, and the results are stronger. Both are patterned after the nonequivariant case (cf. [H, pp. 29-30]):

THEOREM D (THEOREM 4.2). Choose any $\mathrm{O}_{G}$-module $\underline{M}$ such that $\operatorname{proj} \operatorname{dim} \underline{M} \leq 1$.

Then

(1) Moore $G$-spaces of type $(\underline{M}, n)$ exist and are unique up to $G$-equivalence.

(2) Let $X$ be a Moore $G$-complex of type $(\underline{M}, n)$ and $Y$ any $G$-space. Then there is a short-exact sequence of groups (abelian groups, if $n>2$ )

$$
\operatorname{Ext}^{1}\left(\underline{M}, \underline{\pi}_{n+1} Y\right) \mapsto[X, Y] \rightarrow \operatorname{Hom}\left(\underline{M}, \underline{\pi}_{n} Y\right) .
$$

A generalization of (2) - an equivariant Federer spectral sequence-is given in [K2].

Assume (1.3) and that $G$ is a cyclic $p$-group. Then, the conclusions of Theorem $\mathrm{D}$ apply to every $O_{G}$-module, because all such have projective dimension $\leq 1$ (see [T3]).

Theorem D can be applied to obtain a result about classical Moore $G$-spaces. 
COROLlaRY E. Let $G$ be any finite group and $M$ any $\mathbf{Z} G$-module with proj $\operatorname{dim} M<\infty$. Then classical Moore $G$-spaces of type $(M, n)$ exist and have unique weak $G$-homotopy type. The same result applies when $M$ is an arbitrary QG-module.

By weak $G$-homotopy type, here, we mean the relation generated by $G$-maps which are weak homotopy equivalences. Existence in this case is well known (cf. $[\mathbf{S w}]$ ), so this corollary contributes only a uniqueness assertion.

At this point, we want to mention some connections with work of Triantafillou [T1] and Rothenberg and Triantafillou [R \& $\mathbf{T}]$ on equivariant minimal models. The homological algebra of $O_{G}$-modules plays a basic role in [T1], and we have profited considerably from the analysis of projective $O_{G}$-modules in that paper. It is possible that some of our results can be reformulated or derived within the minimal model theory of $[\mathbf{T 1}]$ or $[\mathbf{R} \& \mathbf{T}]$, but that machinery is substantially more complex than the direct constructions of $\S 3$. So, for the "stable" results of this paper, we prefer the present approach. For more elaborate, nonstable examples along the lines of Theorem B, or for purposes of classifying Moore $G$-spaces, the machinery of $[\mathbf{T} \mathbf{1}]$ or $[\mathbf{R} \& \mathbf{T}]$ may well be useful.

We now give a description of the organization of this paper and of some subsidiary results that may be of independent interest.

$\S 2$ contains preliminary material: general terminology and constructions involving $O_{G}$-objects, $O_{G}$-sets, $G$-spaces, $O_{G}$-modules, and $O_{G}$-chain complexes. In addition, there are two results that deserve mention. First, for $O_{G}$-chain complexes $\underline{C}, \underline{D}$ ( $\underline{C}$ bounded), we obtain (in 2.2.3) a spectral sequence for $\operatorname{Hom}(\underline{C}, \underline{D})$ exactly analogous to the standard one (e.g., as in [M, pp. 340-342]). Secondly, we extend slightly (in 2.5) a result of Bredon [B, Lemma 6.5, p. II-15] which enables us to select unique $G$-homotopy classes of $G$-maps that satisfy certain algebraic conditions.

$\S 3$ contains our basic results, namely, theorems on realizing certain rational $O_{G^{-}}$ chain complexes, $O_{G}$-chain maps, and $O_{G}$-chain homotopies. These lead to a classification theorem for stable $G$-mapping sets (Corollary 3.6), which when combined with the spectral sequence of 2.2.3, permits some computations (Corollary 3.9). These do not necessarily involve Moore $G$-spaces.

In addition we present an equivariant version (Theorem 3.1) of a realizability theorem of Wall [W, p. 132], which we apply to obtain some of our other results. A general form of this appeared originally in the Diplomarbeit of W. Lück $[\mathbf{L}]$.

$\S 4$ obtains all the theorems described in the above introduction, except Theorem B.

$\S 5$ constructs the example in Theorem B. The construction is quite long and essentially independent of $\S \S 3,4$. Two features of the construction deserve some comment. First, the role played (in Step 5) by our "generalized Hopf invariant" underscores the stable nature of Theorems A, C. Secondly, the appearance of the "obstruction" group $\operatorname{Ext}^{2}(\underline{M}, \underline{M})=\operatorname{Ext}^{2}\left(\underline{M}, \underline{\pi}_{3} L_{2}\right)$ in $\S 5$ is related to the equivariant Federer spectral sequence mentioned in connection with Theorem D. In fact, it was the appearance of such groups in the spectral sequence that first suggested how we might find the desired example.

The author would like to thank J. P. May for some helpful comments. 
2. Preliminaries. Much of the material we present here is explained in more detail in $[\mathbf{B}]$.

2.1. $O_{G}$-objects. $G$ will always denote a finite group. Let $O_{G}$ be the category of canonical orbits of $G$, that is, the category whose objects are the left-coset spaces $G / H, H \leq G$, and whose morphisms are their $G$-maps. An $O_{G}$-object in a category $C$ is a contravariant functor from $O_{G}$ to $C$; an $O_{G}$-map is a natural transformation of $O_{G}$-objects. Given $O_{G}$-objects, $\underline{A}, \underline{B}$ in $C$, the set of $O_{G}$-maps $\underline{A} \rightarrow \underline{B}$ will be denoted $\operatorname{Hom}(\underline{A}, \underline{B})$. The category of all $O_{G}$-objects and $O_{G}$-maps in $C$ will be denoted $O_{G}^{\circ}(C)$.

When $C$ is well known, we may adapt our terminology accordingly. For example, when $C$ is the category Sets of sets and functions or $T o p_{*}$ of pointed spaces and pointed maps, we speak of $O_{G}$-sets or $O_{G}$-spaces, respectively. If $R$ is a ring and $C=\mathcal{M}_{R}$ is the category of (left) $R$-modules and their homomorphisms, then we may speak of $O_{G}-R$-modules. When $R$ is fixed and clearly understood, or when $R=\mathbf{Z}$, we may speak simply of $O_{G}$-modules.

One particularly simple type of $O_{G}$-object in $C$ is obtained by choosing an object $X$ in $C$ and defining a contravariant functor $O_{G} \rightarrow C$ by the rule $\hat{a} \rightarrow \operatorname{id}{ }_{X}$ for every morphism $\hat{a}$ in $O_{G}$. We call such an $O_{G}$-object constant and denote it by the same symbol $X$.

In some cases, this construction has an important generalization, involving the so-called "fixed-point functor" $\Phi$. In particular, suppose that $C$ is a category for which there is a corresponding category $G-C$, whose objects belong to $C$ and are endowed with a (left) $G$-action and whose morphisms are the morphisms of $C$ respecting this action. Any of the specific categories mentioned above is of this type. Then, there is a functor $\Phi: G-C \rightarrow O_{G}^{\circ}(C)$ satisfying $\Phi X(G / H)=X^{H}$, $\Phi(f: X \rightarrow Y)(G / H)=f^{H}: X^{H} \rightarrow Y^{H}$. We may combine $\Phi$ with various standard functors in the following way.

Suppose that $F: C \rightarrow D$ is a (covariant) functor, and use the same name for the functor $O_{G}^{\circ}(C) \rightarrow O_{G}^{\circ}(D)$ induced by composition with $F$. We then obtain a composite

$$
G-C \stackrel{\Phi}{\rightarrow} O_{G}^{\circ}(C) \stackrel{F}{\rightarrow} O_{G}^{\circ}(D),
$$

which we denote by $\underline{F}$. For example, when $C$ is $T o p_{*}$ and $F$ is the reduced (singular) homology functor $\tilde{H}_{n}: T o p_{*} \rightarrow \mathcal{M}_{\mathbf{z}}$, then $\underline{\tilde{H}}_{n}$ is the homology functor used in the introduction. Similarly if $F$ equals the homotopy functor $\pi_{n}$.

For other examples, $C$ may consist of pointed $C W$ complexes and cellular maps and $F$ may be the functor taking each $C W$ complex to its $n$-skeleton, or $F$ may be the reduced cellular chain complex functor $\tilde{C}_{*}$. Or, let $C=$ Sets, and let $F:$ Sets $\rightarrow$ $\mathcal{M}_{R}$ denote the free-module functor (which, in fact, we will denote by $F$ ). Then, if $X$ is a $G$-set, we write $F \circ \Phi X=\underline{F}[X]$ and call it the free $O_{G}$-module on the $G$-set $X$ (or on the basis $X$ ). If $X$ indexes the $n$-cells of a $G$-complex (resp., rational $G$ complex) $K$, then there is an isomorphism of $O_{G}$-Z-modules (resp., $O_{G}-\mathbf{Q}$-modules) $\underline{\tilde{C}}_{n} K \approx \underline{F}[X]$. Bredon shows that $\underline{F}[X]$ is projective as an $O_{G}-R$-module, for $R=\mathbf{Z}$ $[\mathbf{B}$, p. I-23]. The same result and argument hold for any ring $R$, as do the fact and proof that $O_{G}^{\circ}\left(\mathcal{M}_{R}\right)$ has sufficiently many projectives $[\mathbf{B}$, pp. I-26, 27].

2.2. $O_{G}$-chain complexes. Since the category $O_{G}^{\circ}\left(\mathcal{M}_{R}\right)$ is clearly an abelian category, there is a corresponding category of chain complexes in $O_{G}^{\circ}\left(\mathcal{M}_{R}\right)$, denoted 
$C h\left(O_{G}^{\circ}\left(\mathcal{M}_{R}\right)\right)$. Alternatively, this last may be regarded as the category of $O_{G}$-objects and $O_{G}$-maps in $C h\left(\mathcal{M}_{R}\right)$.

2.2.1. The usual terminology for chain complexes applies here. Thus, chain maps, chain homotopies, and chain equivalences have their usual meaning. We write $\underline{C} \simeq \underline{D}$ to signify that $\underline{C}$ is chain equivalent to $\underline{D}$. If $\underline{C}, \underline{D}$ are two chain complexes, $[\underline{C}, \underline{D}]$ denotes the abelian group of chain maps $\underline{C} \rightarrow \underline{D}$ modulo the relation of chain homotopy.

A chain complex $\underline{C}$ is (i) free, (ii) projective, (iii) bounded, (iv) finite-dimensional, (v) $k$-trivial, provided that each $\underline{C}_{i}$ is (i) free, (ii) projective, (iii) 0 , for $|i|$ sufficiently large, (iv) 0 , for $i$ sufficiently large, (v) 0 , for $i \leq k$, respectively. The full subcategory of all $k$-trivial chain complexes will be denoted $C h^{k}\left(O_{G}^{\circ}\left(\mathcal{M}_{R}\right)\right)$. We shall be particularly interested later in $C h^{1}\left(O_{G}^{\circ}\left(\mathcal{M}_{R}\right)\right)$.

If $\underline{M}$ is an $O_{G}$-module, and $n \in \mathbf{Z}$, let $\underline{M}(n)$ denote the chain complex which is $\underline{M}$ concentrated in degree $n$. A degree $n$ resolution of $\underline{M}$ is an $(n-1)$-trivial chain complex $\underline{C}$, together with a homology equivalence $\underline{C} \rightarrow \underline{M}(n)$. When $n=0$, we may omit reference to $n$ or to degree.

The suspension of a chain complex $\underline{C}$, written $\sum \underline{C}$ is defined by

$$
\left(\sum \underline{C}\right)_{i}=\underline{C}_{i-1}, \quad \partial_{i}\left(\sum \underline{C}\right)=-\partial_{i-1}(\underline{C}) .
$$

2.2.2. The connectivity (resp., dimension) of a chain complex $\underline{C}$, written $\operatorname{conn} \underline{C}$ (resp., $\operatorname{dim} \underline{C}$ ) is defined by

$$
\begin{aligned}
\operatorname{conn} \underline{C} & =\sup \left\{n \mid H_{i} \underline{C}=0, i \leq n\right\}, \\
\operatorname{dim} \underline{C} & =\sup \left\{n \mid \underline{C}_{n} \neq 0\right\} .
\end{aligned}
$$

The homotopy-dimension of $\underline{C}$ is defined only for $\underline{C}$ free, and it is defined relative to some chosen class $\Omega$ of free chain complexes containing $\underline{C}$. The definition is

$$
\operatorname{hodim}_{\Omega} \underline{C}=\inf \{\operatorname{dim} \underline{D} \mid \underline{C} \simeq \underline{D} \text { and } \underline{D} \in \Omega\},
$$

and, in general, the value varies with $\Omega$. We omit reference to $\Omega$ when there is no possibility of confusion. In our applications $\Omega$ will usually consist of the free, 1 trivial chain complexes, i.e., the free chain complexes in $C h^{1}\left(O_{G}^{\circ}\left(\mathcal{M}_{R}\right)\right.$ ) (cf. Corollary $3.2)$.

The projective dimension (resp., free dimension) of an $O_{G}$-module $\underline{M}$, written proj $\operatorname{dim} \underline{M}$ (resp., fr $\operatorname{dim} \underline{M}$ ) is, as usual, the minimum length of all projective (resp., free) resolutions of $\underline{M}$. It is easy to see that $\operatorname{proj} \operatorname{dim} \underline{M}=\operatorname{fr} \operatorname{dim} \underline{M}$ except when $\underline{M}$ is projective but not free, in which case, by Eilenberg's trick, fr $\operatorname{dim} \underline{M}=1$.

Let $l(G)$ denote the maximum $l$ for which there exists a sequence of proper inclusions of subgroups of the form

$$
e=G_{0}<G_{1}<\cdots<G_{l-1}<G_{l}=G .
$$

Since $\left[G_{i}: G_{i+1}\right] \geq 2$, it follows that $l(G) \leq \log _{2}|G|$.

LEMMA [R \& $\mathbf{T}]$. If $\underline{M} \in \mathcal{O}_{G}^{\circ}\left(\mathcal{M}_{Q}\right)$, then $\operatorname{proj} \operatorname{dim} M \leq l(G)$.

2.2.3. A spectral sequence for $\left[\sum^{k} \underline{C}, \underline{D}\right]$. Let $\underline{C}, \underline{D}$ belong to $C h\left(O_{G}^{\circ}\left(\mathcal{M}_{R}\right)\right)$, and define a chain complex $\operatorname{Hom}(\underline{C}, \underline{D})$ in $C h\left(\mathcal{M}_{R}\right)$, exactly as in $[\mathbf{M}]$, by

$$
\operatorname{Hom}(\underline{C}, \underline{D})_{r}=\prod_{i \in \mathbf{Z}} \operatorname{Hom}\left(\underline{C}_{i}, \underline{D}_{i+r}\right)
$$




$$
\partial_{r}(H \circ m)\left(\underline{f}_{i}\right)=\left(\underline{g}_{i}\right), \quad \text { where } \underline{g}_{i}=\underline{\partial}_{i+r}(D) \circ \underline{f}_{i}+(-1)^{r-1} \underline{f}_{i-1} \circ \underline{\partial}_{i}(C) .
$$

Our interest in $\operatorname{Hom}(\underline{C}, \underline{D})$ is due to the well-known observation [M, p. 44] that

$$
[\underline{C}, \underline{D}]=H_{0}(\operatorname{Hom}(\underline{C}, \underline{D})) \text {. }
$$

In fact, it is not hard to check that for every $k \in \mathbf{Z}$,

$$
\left[\sum^{k} \underline{C}, \underline{D}\right]=H_{k}(\operatorname{Hom}(\underline{C}, \underline{D})) .
$$

We shall interpret the well-known spectral sequence for $H_{*}(\operatorname{Hom}(\underline{C}, \underline{D}))[\mathbf{M}, \mathrm{pp}$. 340-342] as converging to $\left[\sum^{k} \underline{C}, \underline{D}\right]$ via the above. Carlsson [C2] has also made use of this in another context.

If $\underline{M}$ is an $O_{G}$-module, then let $H_{G}^{r}(\underline{C} ; \underline{M})$ denote $H_{-r}(\operatorname{Hom}(\underline{C}, \underline{M}(0)))$. This is just the algebraic version of Bredon cohomology $[\mathbf{B}]$.

Proposition. Suppose $\underline{C}, \underline{D} \in \operatorname{Ch}\left(\mathcal{O}_{G}^{\circ}\left(\mathcal{M}_{R}\right)\right)$ with $\underline{C}$ bounded. There exists a spectral sequence $E_{p q}^{r}$ such that

(a) $E_{p q}^{2}=H_{G}^{-p}\left(\underline{C} ; H_{q} \underline{D}\right)$. Thus, by $[\mathbf{B}, p . \mathrm{I}-24]$, if $\underline{C}$ is a degree $n$ resolution, then $E_{p q}^{2}=\mathrm{Ext}^{-p-n}\left(H_{n} \underline{C}, H_{q} \underline{D}\right)$, where $\mathrm{Ext}^{i}$ is the usual ith derived functor of Hom on $O_{G}^{\circ}\left(\mathcal{M}_{R}\right)$.

(b) If $\underline{C}, \underline{D}$ are nonnegative, then $E_{p q}^{r}$ is a second-quadrant spectral sequence.

(c) $E_{p q}^{r}$ converges to the bigraded module associated to some dimensionwise-finite filtration of $\left[\sum^{n} \underline{C}, \underline{D}\right], n \in \mathbf{Z}$.

REMARK. The proof is the standard double-complex argument, as it appears, say, in [M, pp. 340-342]. The double complex is given by $K_{p q}=\operatorname{Hom}\left(\underline{C}_{-p}, \underline{D}_{q}\right)$, with differentials induced by those of $\underline{C}$ and $\underline{D}$. The boundedness of $\underline{C}$ implies that the total complex Tot $K$ equals $\operatorname{Hom}(\underline{C}, \underline{D})$ and that the standard (first) filtration is finite.

2.3. $G$-spaces. We sharpen our earlier usage and, henceforth, let $T o p_{*}$ denote the category of pointed, compactly-generated Hausdorff spaces and pointed, continuous maps. All spaces and maps considered in this paper will belong to $T o p_{*}$.

If $\tau_{*}$ is a subcategory of $T o p_{*}$, let $G-\tau_{*}$ consist of all $G$-spaces $X$ and $G$-maps in $\tau_{*}$ such that each fixed-point subspace $X^{H}$ is in $\tau_{*}$. A similar convention applies to pairs of spaces.

We shall be primarily interested in subcategories $\mathcal{W}_{*}^{n}$ (resp., $\mathbf{Q} \mathcal{W}_{*}^{n}$ ) of $T o p_{*}$, consisting of $\mathrm{CW}$ complexes (resp., rational CW complexes) $K$ and cellular maps such that the $n$-skeleton $K^{n}$ is a point. More specifically, we shall focus on $\mathcal{W}_{*}^{1}, \mathbf{Q} \mathcal{W}_{*}^{1}$, and the corresponding categories $G-\mathcal{W}_{*}^{1}$ and $G-\mathbf{Q} \mathcal{W}_{*}^{1}$. These are all categories of $G$ complexes in the sense of $[\mathbf{B}, \mathbf{W n}]$. In order to deal with both cases simultaneously, we sometimes write $\mathbf{Z} \mathcal{W}_{*}^{1}$ for $\mathcal{W}_{*}^{1}$ and then $R \mathcal{W}_{*}^{1}$, with $R=\mathbf{Z}$ or $\mathbf{Q}$.

It is worth pointing out here that we consider rational $\mathrm{CW}$ complexes to be built inductively out of rational cells which are cones on (fixed models of) rational spheres. The dimension of such a complex is, as usual, the supremum of the dimensions of its cells, and the dimension of each cell is one greater than that of the corresponding sphere. Our convention will be to refer to the homology-dimension of a rational sphere as its dimension and to write $S^{n}$ both for the ordinary $n$ dimensional sphere and for the rational one. (As a $\mathrm{CW}$ complex, of course, the rational $S^{n}$ has dimension $\geq n+1$, but as a rational space, by our convention, it 
has dimension $n$.) The context will make our usage clear, but for the most part in $\S \S 3-5$ we will be dealing with rational complexes. All these remarks carry over, of course, to the corresponding categories $G-\mathbf{Q} \mathcal{W}_{*}^{n}$.

In any of the above categories $G$ - $T_{*}$ we use $\sim$ to denote $G$-homotopy and $\simeq$ to denote $G$-homotopy-equivalence ( $G$-equivalence, for short). For $G$-spaces $X, Y$ in $G-T_{*},[X, Y]$ will denote the set of $G$-homotopy classes of $G$ maps $X \rightarrow Y$, all taken to be in $\tau_{*}$. If $X$ is a (double) suspension, then $[X, Y]$ has a natural (abelian) group structure, by the standard argument. Similarly for maps of pairs.

Let $\mathcal{V}$ be a class of spaces in $G-\mathcal{W}_{*}$ and choose $X$ in $\mathcal{V}$. Define

$$
\operatorname{hodim} \mathcal{V} X=\inf \{\operatorname{dim} Y \mid Y \text { in } \mathcal{V} \text { and } Y \simeq X\}
$$

When there can be no confusion, we omit reference to $\mathcal{V}$ and write hodim $X$ even though, in general, hodim $X$ will depend on $\mathcal{V} .^{1}$

Finally, for any $X$ in $G$-Top $p_{*}$, let

$$
\text { conn } X=\sup \left\{n \mid \underline{\pi}_{i} X=0, \text { for } i \leq n\right\} \text {. }
$$

Similarly for pairs of spaces in $G$ - $T o p_{*}$.

2.4. $O_{G}$-sets. The most important examples of $O_{G}$-sets are those of the form $\Phi S$ for $S$ a $G$-set. This is because of the following

\subsubsection{LEMMA. $\Phi S$ is projective.}

By this we mean $\Phi S$ satisfies the usual universal lifting property for projectives. That is, noting that epimorphisms in $O_{G}^{\circ}($ Sets $)$ are precisely those maps $\underline{f}$ satisfying $\underline{f}(G / H)$ is surjective, for all $H \leq G$, we are asserting that every diagram

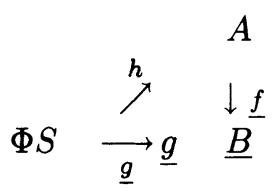

with $\underline{f}$ epi, can be completed.

PROOF. This is a standard argument, similar to that in [B, p. I-23]. It suffices to treat the case $S=G / H$. Then, $\Phi S(G / K)=(G / H)^{K}=\operatorname{Hom}(G / K, G / H)$. For $\gamma$ in this set, define

$$
\underline{h}(\gamma)=\underline{A}(\gamma)\left(a_{0}\right)
$$

where $a_{0} \in \underline{A}(G / H)$ is any chosen lift of $\underline{g}(G / H)\left(\operatorname{id}_{G / H}\right)$.

It is possible to show that every projective $O_{G}$-set is of the form $\Phi S$, but we do not need this fact here.

There is a functor ${ }^{+}:$Sets $\rightarrow$ To $p_{*}$, obtained by adjoining a disjoint basepoint. This induces corresponding functors ${ }^{+}: G$-Sets $\rightarrow G$-Top $p_{*}$ and ${ }^{+}: O_{G}^{\circ}($ Sets $) \rightarrow$ $O_{G}^{\circ}\left(T o p_{*}\right)$ which are compatible with $\Phi$.

2.5. Extending a lemma of Bredon. We state and prove here a slight extension of Lemma 6.5 , p. II.15 of [B]. The extension is needed when we deal with nonabelian fundamental group (as in the proof of 4.2 and in 5.4).

\footnotetext{
${ }^{1}$ For example, if $M$ is a $\mathrm{ZG}$-module that is projective but not free, there exists a 3-dimensional classical Moore $G$-space of type $(M, 3)$ in $G-\mathcal{W}_{*}^{1}$, but all $G$-equivalent complexes in $G-\mathcal{W}_{*}^{2}$ are at least 4-dimensional.
} 
Recall (2.1) that the usual path-component functor $\pi_{0}:$ Top $\rightarrow$ Sets determines a functor

$$
G-T o p_{*} \stackrel{\Phi}{\rightarrow} O_{G}^{\circ}\left(T o p_{*}\right) \stackrel{\pi_{0}}{\rightarrow} O_{G}^{\circ}(S e t s)
$$

which we denote by $\underline{\pi}_{0}$.

2.5.1. Lemma. Let $S$ be a $G$-set and $Y$ a pointed $G$-space. The natural map

$$
\left[S^{+}, Y\right] \rightarrow \operatorname{Hom}\left(\Phi S, \underline{\pi}_{0} Y\right)
$$

is bijective.

ProOF. It suffices to treat the case $S=G / H$.

The left-hand side is obtained from $\operatorname{Hom}\left(S^{+}, Y\right)=\operatorname{Hom}\left(G / H^{+}, Y\right)$ by passing to $G$-homotopy classes. But there is a standard isomorphism $\operatorname{Hom}\left(G / H^{+}, Y\right) \approx Y^{H}$ under which $G$-homotopy classes go to path-components. Thus, the left-hand side is $\pi_{0}\left(Y^{H}\right)$.

The right-hand side may be evaluated by observing that $\Phi S(G / K)=(G / H)^{K}=$ $\operatorname{Hom}(G / K, G / H)$, as in 2.4.1. It follows that an element $f \in \operatorname{Hom}\left(\Phi S, \underline{\pi}_{0} Y\right)$ may be completely specified by the value $\underline{f}(G / H)\left(\operatorname{id}_{G / H}\right) \in \underline{\pi}_{0} \bar{Y}(G / H)=\pi_{0}\left(Y^{H}\right)$. That is, the right-hand side also equals $\pi_{0}\left(Y^{H}\right)$. It is not hard to see that the natural map identifies these.

2.5.2. Corollary. Let $X$ be a $G$-set, $(L, K)$ a pair of pointed $G$-spaces, and $n$ an integer $\geq 1$. Then, there are natural bijections

(a) $\left[S^{n-1} \wedge X^{+}, L\right] \approx \operatorname{Hom}\left(\Phi X, \underline{\pi}_{n-1} L\right)$,

(b) $\left[\left(D^{n}, S^{n-1}\right) \wedge X^{+},(L, K)\right] \approx \operatorname{Hom}\left(\Phi X, \underline{\pi}_{n}(L, K)\right)$.

Proof. (a) Apply the lemma to the $G$-space $Y=L^{S^{n-1}}$ and use the standard bijection

$$
\operatorname{Hom}\left(X^{+}, Y\right) \approx \operatorname{Hom}\left(S^{n-1} \wedge X^{+}, L\right),
$$

which respects homotopy classes.

(b) Similar to (a).

REMARKS. (a) Note that when $n \geq 3$, we have

$$
\begin{aligned}
\operatorname{Hom}\left(\Phi X, \underline{\pi}_{n-1} L\right) & \approx \operatorname{Hom}\left(\underline{F}[X], \underline{\pi}_{n-1}, L\right), \\
\operatorname{Hom}\left(\Phi X, \underline{\pi}_{n}(L, K)\right) & \approx \operatorname{Hom}\left(\underline{F}[X], \underline{\pi}_{n}(L, K)\right) .
\end{aligned}
$$

Bredon's Lemma 6.5, op. cit., is essentially 2.5.2(a), for $n \geq 3$, with the first righthand expression replacing the one on the left.

(b) We shall be applying the corollary in the context of rational complexes. In that case, we always interpret $S^{n-1}, n \geq 2$, as a rational $(n-1)$-sphere, and $D^{n}$ as the cone $\hat{c} S^{n-1}$. Because we are using compactly-generated topologies, this modification does not affect the above proofs.

3. Realizing chain complexes and chain maps. Throughout this section, we shall confine spaces and maps to the categories $R W_{*}^{1}$ and $G-R W_{*}^{1}, R=\mathbf{Z}$ or $\mathbf{Q}$ (see 2.3). Corresponding algebraic restrictions will be in force. Thus, $R$ will always mean $\mathbf{Z}$ or $\mathbf{Q}$, and chain complexes will always belong to $C h^{1}\left(\mathcal{M}_{R}\right)$ or $C h^{1}\left(O_{G}^{\circ}\left(\mathcal{M}_{R}\right)\right)$, i.e., they will be 1-trivial (see 2.2.1).

Proofs of the main theorems are given at the end of the section. 
In general, we shall use the terms "realizing," "realization," etc. in connection with geometric objects, such as $G$-complexes or cellular $G$-maps, for which certain corresponding algebraic objects have been prescribed. The context should make this usage clear.

3.1. THEOREM. Suppose that $L \in G-R \mathcal{W}_{*}^{1}$ and that $\underline{C}$ is a free chain complex in $C h^{1}\left(\mathcal{O}_{G}^{\circ}\left(\mathcal{M}_{R}\right)\right)$. Let $\phi: \underline{C} \rightarrow \underline{\tilde{C}}_{*} L$ be a homology equivalence. Then, there exist a $K \in G-R \mathcal{W}_{*}^{1}$ and a cellular $G$-map $f: K \rightarrow L$ realizing $\phi$.

REMARK. This theorem (in somewhat different form) was originally discovered by W. Lück $[\mathbf{L}]$ and was rediscovered independently by the author. It is an equivariant version (with some simplifying assumptions, here, which are suited to this context) of a theorem of Wall [W, p. 132].

The following consequence of 3.1, although interesting in its own right, will be used here only for the formulation of some of our results below and in $\S 4$. We refer the reader to 2.2 .2 and 2.3 , in which definitions of hodim are given for chain complexes and for spaces, respectively. The classes $\Omega$ and $\mathcal{V}$ in those definitions are understood, here, to refer to all free chain complexes in $C h^{1}\left(O_{G}^{\circ}\left(\mathcal{M}_{R}\right)\right)$ and all spaces in $G-R W_{*}^{1}$, respectively.

3.2. Corollary. For any $K \in G-R \mathcal{W}_{*}^{1}$, hodim $\underline{\tilde{C}}_{*} K=\operatorname{hodim} K$.

PROOF. By $G$-cellular approximation [B, II.12], ${ }^{2} K \simeq L$ implies $\underline{\tilde{C}}_{*} K \simeq \underline{\tilde{C}}_{*} L$. Using an $L \simeq K$ such that hodim $K=\operatorname{dim} L$, we obtain

$$
\operatorname{hodim} \underline{\tilde{C}}_{*} K \simeq \operatorname{hodim} \underline{\tilde{C}}_{*} L \leq \operatorname{dim} \underline{\tilde{C}}_{*} L=\operatorname{dim} L=\operatorname{hodim} K .
$$

For the reverse inequality, we choose a free $\underline{C} \in C h^{1}\left(\mathcal{O}_{G}^{\circ}\left(\mathcal{M}_{R}\right)\right)$ and a chain equivalence $\Phi: \underline{C} \rightarrow \underline{\tilde{C}}_{*} K$ with $\operatorname{dim} \underline{C}=\operatorname{hodim} \underline{\tilde{C}}_{*} K$. We apply 3.1 to obtain a $G$-map $f: L^{\prime} \rightarrow K$ realizing $\phi$. The Whitehead Theorem for $G$-complexes [B, II.12] implies that $f$ is a $G$-equivalence. Thus, hodim $K=\operatorname{hodim} L^{\prime} \leq \operatorname{dim} L^{\prime}=\operatorname{dim} \underline{C}=$ $\operatorname{hodim} \underline{\tilde{C}}_{*} K$.

Theorem 3.1 asserts a type of relative realizability. In our next result, the realizability is absolute. We refer the reader to 2.2 .2 and 2.3 to recall the definition of conn and conn, respectively.

3.3. THEOREM. Suppose $\underline{C}$ is a free chain complex in $C h^{1}\left(O_{G}^{\circ}\left(\mathcal{M}_{\mathbf{Q}}\right)\right)$ such that $\operatorname{hodim} \underline{C} \leq 2 \operatorname{conn} \underline{C}+2$.

Then $\underline{C}$ has a realization. In particular, every bounded, free $\underline{C}$ in $C h^{1}\left(\mathcal{O}_{G}^{\circ}\left(\mathcal{M}_{\mathbf{Q}}\right)\right)$ has a stable realization.

We now turn to chain maps and homotopies.

3.4. THEOREM. Let $(L, K)$ be a pair of $G$-complexes and $f^{\prime}: K \rightarrow M a$ cellular $G$-map, with $K, L, M \in G-\mathbf{Q} \mathcal{W}_{*}^{1}$ and

$$
\operatorname{dim}(L \backslash K) \leq 2 \underline{\text { conn }} M+1 .
$$

Suppose that $\phi: \underline{\tilde{C}}_{*} L \rightarrow \tilde{\tilde{C}}_{*} M$ is a chain map extending $\underline{\tilde{C}}_{*} f^{\prime}: \underline{\tilde{C}}_{*} K \rightarrow \underline{\tilde{C}}_{*} M$. Then there exists a cellular $G$-map $f: L \rightarrow M$ realizing $\phi$ and extending $f^{\prime}$.

\subsection{Corollary. Let $K, L \in G-\mathbf{Q} W_{*}^{1}$.}

\footnotetext{
${ }^{2}$ This works for rational complexes as well.
} 
(a) Suppose $\operatorname{dim} K \leq 2 \underline{\text { conn }} L+1$. Then every chain map $\underline{\tilde{C}}_{*} K \rightarrow \tilde{C}_{*} L$ has a realization.

(b) Suppose $\operatorname{dim} K \leq 2$ conn $L$. Then cellular $G$-maps $K \rightarrow L$ are $G$-homotopic provided that the induced chain maps are chain homotopic.

Proof. (a) This follows immediately from 3.4 taking $K$ in 3.4 to be *.

(b) A chain homotopy between chain maps $\underline{\tilde{C}}_{*} K \rightarrow \underline{\tilde{C}}_{*} L$ may be interpreted as a chain map $\underline{\tilde{C}}_{*}\left(I^{+} \wedge K\right) \rightarrow \underline{\tilde{C}}_{*} L$. Apply 3.4 to this.

Note that the converse of 3.5 (b) holds without dimension restrictions as in the proof of 3.2. Thus, we get

3.6. COROLlary. Suppose that $K, L \in G-\mathbf{Q} \mathcal{W}_{*}^{1}$. Then, the natural map $[K, L] \rightarrow\left[\underline{\tilde{C}}_{*} K, \underline{\tilde{C}}_{*} L\right]$ is well defined and

(a) surjective, if hodim $K \leq 2$ conn $L+1$, and

(b) bijective, if hodim $K \leq 2$ conn $L$.

PRoOF. If, in the hypotheses, hodim $K$ is replaced by $\operatorname{dim} K$, then (a) and (b) are immediate consequences of 3.5 and the subsequent remark. It remains to observe that neither the domain nor the range of the map in question are affected essentially when $K$ is replaced by a $G$-equivalent complex.

3.7. Corollary. Suppose that $\underline{C}$ is a free chain complex in $C h^{1}\left(O_{G}^{\circ}\left(\mathcal{M}_{\mathbf{Q}}\right)\right)$.

(a) If hodim $\underline{C} \leq 2 \underline{\text { conn }} C+1$, then any two realizations of $\underline{C}$ are $G$-equivalent.

(b) If hodim $\underline{C}<\infty$, then any two stable realizations of $\underline{C}$ are stably $G$ equivalent.

Proof. Of course, (b) follows from (a). To obtain (a), apply 3.2 and 3.6(a) to two realizations $K, L$, obtaining a cellular $G$-map $K \rightarrow L$ that induces a chain equivalence $\underline{\tilde{C}}_{*} K \rightarrow \underline{\tilde{C}}_{*} L$. Now apply the Whitehead Theorem for $G$-complexes.

3.8. REMARK. Set

$$
\{K, L\}=\operatorname{colim}\left[\sum^{l} K, \sum^{l} L\right] .
$$

As usual this has a natural abelian-group structure. Assume that $K, L \in \mathbf{Q} \mathcal{W}_{*}^{1}$ and hodim $K<\infty$. Corollary 3.6 implies that there is a bijection $\{K, L\} \approx\left[\underline{\tilde{C}}_{*} K, \underline{\tilde{C}}_{*} L\right]$ and that the natural map $[K, L] \rightarrow\{K, L\}$ is

(a) surjective, if hodim $K \leq 2$ conn $L+1$, and

(b) bijective, if hodim $K \leq 2$ conn $L$.

One checks easily that the bijection is an abelian-group isomorphism. We now combine this remark with the spectral sequence of 2.2.3.

3.9. Corollary. Choose $K, L \in G-\mathbf{Q} W_{*}^{1}$ with hodim $K<\infty$. There is a second-quadrant spectral sequence $E_{p q}^{r}$ converging to $\left\{\sum^{n} K, L\right\}, n \in \mathbf{Z}$, such that

(a) $E_{p q}^{2}=\tilde{H}_{G}^{-p}\left(K ; \underline{\tilde{H}}_{q} L\right)$ (Bredon cohomology $\left.[\mathbf{B}]\right)$;

(b) if $K$ is a Moore $G$-space of type $(\underline{M}, n)$, then $E_{p q}^{2}=\operatorname{Ext}^{-p-n}\left(M, \underline{\tilde{H}}_{q} L\right)$.

We now turn to proofs of 3.1, 3.3, 3.4. Each of these follows a similar, familiar pattern. Each involves a construction which is inductive on skeleta and begins trivially. In each, the inductively assumed algebraic data is used to produce a map of $O_{G}$-modules of the form $\underline{F}[X] \rightarrow \underline{\pi}, \underline{\pi}$ some homotopy group, which is 
converted to a certain $G$-map by means of Corollary 2.5.2. The $G$-map is then used to complete the inductive step. We describe only the inductive step in what follows.

Proof of TheOREM 3.1. Assume that $K^{n-1}$, the $(n-1)$-skeleton of $K$, and $f^{n-1}: K^{n-1} \rightarrow L$ have been defined realizing $\phi$ through dimension $n-1$. Consider the following diagram, in which the middle horizontal rows are exact.

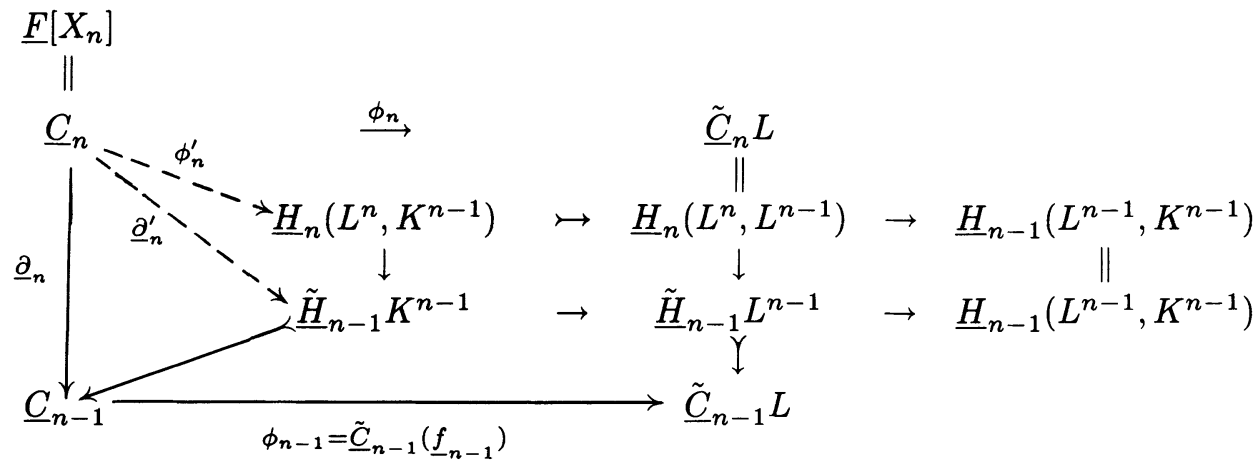

(We abuse notation slightly here, since $f^{n-1}$ is not an inclusion.) Note that $\underline{\partial}_{n}\left(\underline{C}_{n}\right) \subset \operatorname{ker} \underline{\partial}_{n-1}$, and ker $\underline{\partial}_{n-1}$ can be identified with $\underline{\tilde{H}}_{n-1} K^{n-1}$. Thus, $\underline{\partial}_{n}^{\prime}$ exists and is unique, and the commutativity of the diagram then implies that $\operatorname{im} \phi_{n} \subset \underline{H}_{n}\left(L^{n}, K^{n-1}\right)$, i.e., $\phi_{n}^{\prime}$ exists and is unique. Finally, the inductive hypothesis implies that $\underline{\operatorname{conn}}\left(L^{n}, K^{n-1}\right) \geq n-1$, so that we may identify $\underline{\pi}_{n}\left(L^{n}, K^{n-1}\right)$ and $\underline{H}_{n}\left(L^{n}, K^{n-1}\right)$.

In summary, then, we have a composite

$$
\underline{F}\left[X_{n}\right] \hookrightarrow \underline{C}_{n} \stackrel{\phi_{n}^{\prime}}{\rightarrow} \underline{H}_{n}\left(L^{n}, K^{n-1}\right)=\underline{\pi}_{n}\left(L^{n}, K^{n-1}\right) .
$$

By Corollary 2.5.2, this may be represented by a $G$-map, unique up to $G$-homotopy, $\left(D^{n}, S^{n-1}\right) \wedge X_{n}^{+} \rightarrow\left(L^{n}, K^{n-1}\right)$. More accurately, we obtain a commutative diagram of $G$-maps

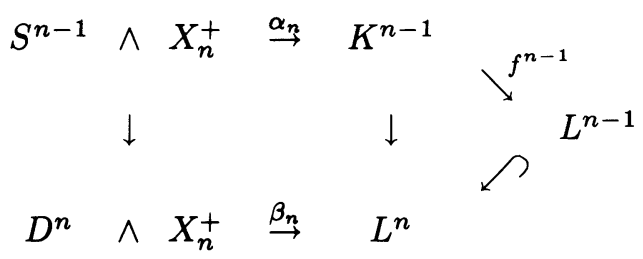

Define $K^{n}$ to be the pushout of

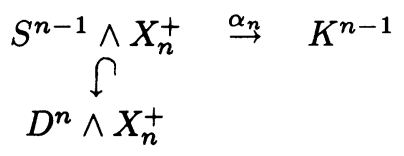

and use $\beta_{n}$ to extend $f^{n-1}$ to a $G$-map $f^{n}: K^{n} \rightarrow L^{n}$. One checks easily that $f^{n}$ has the required properties.

The proofs of 3.3 and 3.4 require the following lemma, which ig a standard (and easily verified) fact in ordinary homotopy theory. 
3.10. LemMA. Suppose $K \in \mathbf{Q} W^{1}$. The Hurewicz map $\pi_{i} K \rightarrow H_{i} K$ is bijective if $i \leq 2$ conn $K$ and surjective if $i \leq 2 \operatorname{conn} K+1$.

Proof of Theorem 3.3. Write $\underline{C}_{i}=\underline{F}\left[X_{i}\right], i \geq 1$, for $G$-sets $X_{i}$. Suppose that $L_{i}$ have been constructed, for $i<n$, with each $L_{i}$ obtained from $L_{i-1}$ by attaching $D^{i} \wedge X_{i}^{+}$, such that when $\underline{H}_{i}\left(L_{i}, L_{i-1}\right)=\underline{\tilde{C}}_{i} L_{i}$ is accordingly identified with $\underline{C}_{i}$, the squares

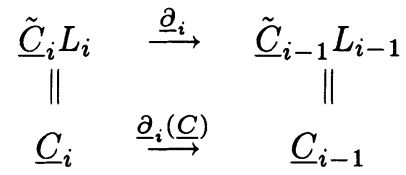

commute.

It follows that $\underline{\tilde{H}}_{n-1} L_{n-1}$ may be identified with ker $\underline{\partial}_{n-1}(\underline{C})$ and we obtain a diagram

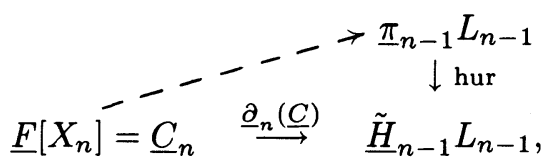

where hur is the Hurewicz map.

Suppose that hur is an epimorphism (i.e., $\operatorname{hur}(G / H)$ is surjective for each $H \leq$ $G$ ). Since $\underline{F}\left[X_{n}\right]$ is projective, the diagram can be completed, and, by Corollary 2.5.2, the lift $\underline{F}\left[X_{n}\right] \rightarrow \underline{\pi}_{n-1} L_{n-1}$ is represented by a $G$-map $S^{n-1} \wedge X_{n}^{+} \rightarrow L_{n-1}$, which we denote $d_{n}$. Then, we get $L_{n}=\hat{c}\left(d_{n}\right)$. It is straightforward to check that $(3.11 \mathrm{n})$ is satisfied.

It remains to determine when hur is epi. By 3.10, this occurs as long as $n-1 \leq 2$ conn $L_{n-1}+1$. There are two cases. (1) $n-1 \leq \operatorname{conn} \underline{C}$, in which case $\underline{\operatorname{conn}} L_{n-1} \geq n-2$. (2) $n-1>\operatorname{conn} \underline{C}$, in which case conn $L_{n-1}=\operatorname{conn} \underline{C}$. In the first case, the desired inequality is always satisfied. In the second, it is satisfied for $n-1 \leq 2 \operatorname{conn} \underline{C}+1$. Thus, the above construction can be continued until we obtain $L_{n}$ for $n=2 \operatorname{conn} \underline{C}+2$. But by 3.1 and the hypothesis, we may assume $\underline{C}$ satisfies $\operatorname{dim} \underline{C} \leq 2 \operatorname{conn} \underline{C}+2$, and so we are done.

PROOF OF THEOREM 3.4. For each $i \geq 2$, let $W_{i}$ be a $G$-set indexing the $i$-cells of $L \backslash K$. Clearly, $\underline{\tilde{C}}_{i} L=\underline{\tilde{C}}_{i} K \oplus \underline{F}\left[W_{i}\right], i \geq 2$.

The desired extension of $f^{\prime}$ to $f^{2}: L^{2} \cup K \rightarrow M$ is straightforward and will be left to the reader. Suppose then that we have been able to extend $f^{\prime}$ to a cellular $G$-map $f^{n-1}: L^{n-1} \cup K \rightarrow M, n-1 \geq 2$, so as to induce $\phi$ on $\underline{C}_{i} L$ for $i \leq n-1$. We have the following commutative diagram

$$
\begin{aligned}
& \underline{F}\left[W_{n}\right] \subset \underline{\tilde{C}}_{n} L \quad \rightarrow \quad \underline{Z}_{n-1} L=\underline{H}_{n-1} L^{n-1} \\
& \phi \downarrow \quad \phi \mid \downarrow \quad \downarrow f_{*}^{n-1} \\
& \underline{\tilde{C}}_{n} M \quad \rightarrow \quad \underline{Z}_{n-1} M=\underline{H}_{n-1} M^{n-1} \\
& \underline{\pi}_{n}\left(M^{n}, M^{n-1}\right)
\end{aligned}
$$

Use Corollary 2.5.2 to choose a $G$-map $\left(D^{n}, S^{n-1}\right) \wedge W_{n}^{+} \stackrel{\hat{f}}{\rightarrow}\left(M^{n}, M^{n-1}\right)$ representing

$$
\underline{F}\left[W_{n}\right] \subset \underline{\tilde{C}}_{n} L \stackrel{\phi}{\rightarrow} \underline{\tilde{C}}_{n} M=\underline{\pi}_{n}\left(M^{n}, M^{n-1}\right) .
$$


By (3.12) above,

$$
S^{n-1} \wedge W_{n}^{+} \stackrel{\hat{f} \mid}{\rightarrow} M^{n-1}
$$

and

$$
S^{n-1} \wedge W_{n}^{+} \stackrel{d_{n}}{\rightarrow} L^{n-1} \stackrel{f^{n-1}}{\rightarrow} M^{n-1}
$$

induce the same homology homomorphism

$$
\underline{F}\left[W_{n}\right]=\underline{H}_{n-1}\left(S^{n-1} \wedge W_{n}^{+}\right) \rightarrow \underline{H}_{n-1} M^{n-1},
$$

with $d_{n}$ the attaching map for $D^{n} \wedge W_{n}^{+}$.

Now assume that hur : $\underline{\pi}_{n-1} M^{n-1} \rightarrow \underline{H}_{n-1} M^{n-1}$ is an isomorphism. It follows that both of the above maps (3.13) and (3.14) induce the same homotopy homomorphisms

$$
\underline{F}\left[W_{n}\right]=\underline{\pi}_{n-1}\left(S^{n-1} \wedge W_{n}^{+}\right) \rightarrow \underline{\pi}_{n-1} M^{n-1} .
$$

By Corollary 2.5.2 again, it follows that the maps are $G$-homotopic. Thus, by the $G$-Homotopy Extension Theorem [B, p. I-1], $\hat{f}$ is $G$-homotopic to a $G$-map $\bar{f}$ extending $f^{n-1} \circ d_{n}$. If $\chi_{n}: D^{n} \wedge W_{n}^{+} \rightarrow L^{n}$ is the characteristic map extending $d_{n}$, then the desired $G$-map $f^{n}: L^{n} \cup K \rightarrow M$ can be defined as $f^{n-1} \cup\left(\bar{f} \circ \chi_{n}^{-1}\right)$.

It remains to determine when $\underline{\pi}_{n-1} M^{n-1}=\underline{H}_{n-1} M^{n-1}$. This discussion is analogous to that in the previous proof, the result being that we need to require $n-1 \leq 2$ conn $M$. Thus the foregoing construction can continue until we have obtained $f^{n}, n=2$ conn $M+1$.

4. Applications to Moore $G$-spaces. The conventions of $\S 3$ still apply. Spaces belong to $G-R \underline{\mathcal{W}}_{*}^{1}$, modules to $O_{G}^{\circ}\left(\underline{\mathcal{M}}_{R}\right)$, and chain complexes to $C h^{1}\left(\mathcal{O}_{G}^{\circ}\left(\underline{\mathcal{M}}_{R}\right)\right), R=\mathbf{Z}$ or $\mathbf{Q}$ (see 2.3, 2.2.1).

4.1. THEOREM. (a) If $\underline{M} \in \mathcal{O}_{G}^{\circ}\left(\mathcal{M}_{\mathrm{Q}}\right)$ and either each $\underline{M}(G / H)$ is a finitedimensional vector space or $n \geq \operatorname{fr} \operatorname{dim} \underline{M}$, then Moore $G$-spaces of type $(\underline{M}, n)$ exist. (b) Under the assumptions of (a), every degree $n$ free resolution of $\underline{M}$ in $C h^{1}\left(\mathcal{O}_{G}^{\circ}\left(\underline{\mathcal{M}}_{\mathrm{Q}}\right)\right)$ has a realization. (c) Suppose that $\underline{M} \in \mathcal{O}_{G}^{\circ}\left(\underline{\mathcal{M}}_{R}\right), \underline{C}$ is a degree $n$ free resolution of $\underline{M}$ in $C h^{1}\left(O_{G}^{\circ}\left(\underline{\mathcal{M}}_{R}\right)\right)$, and $Y$ is a Moore $G$-space of type $(\underline{M}, n)$. Then there exists a Moore $G$-space realizing $\underline{C}$ and $G$-equivalent to $Y$.

ProOF. (c) By [B, p. II.17], we may assume that $Y$ has no $i$-cells $0<i<n$, which implies that $\tilde{C}_{*} Y$ is a degree $n$ free resolution of $\underline{M}$. Thus, there is a chain equivalence $\underline{C} \rightarrow \underline{\tilde{C}}_{*} Y$, to which we apply Theorem 3.1 and the Whitehead Theorem for $G$-spaces $[\mathbf{B}]$.

(a) When each $\underline{M}(G / H)$ is finite-dimensional, we use a theorem of Triantafillou [T2], together with [B, p. II.17], to find a rational $G$-space with no 1-cells such that $\underline{\text { conn }} Z \geq 1, \underline{\tilde{H}}^{n} Z=\underline{M}^{*}$, and $\underline{\tilde{H}}^{i} Z=0$, for $i \neq n$. Here $\underline{M}^{*}$ is the dual of $\underline{M}$. $Z$ is the desired Moore $G$-space. When $n \geq \operatorname{fr} \operatorname{dim} \underline{M}$, we choose a minimal degree $n$ free resolution of $\underline{M}$ and use Theorem 3.3 to realize it.

(b) This follows immediately from (a) and (c).

Assertion (c) above shows that $G$-homotopy types of Moore $G$-spaces cannot be distinguished via the resolutions they may realize. This is in accord, of course, with the nonequivariant case. 
Our next result specializes to the case of low projective dimension. Here we can say more than in the general case, and we use it as a "best case" illustration. The proof is independent of $\S 3$ and is similar to the well-known, nonequivariant argument of [H, pp. 29-30].

THEOREM 4.2. Choose any $(\underline{M}, n)$ with $\underline{M} \in \mathcal{O}_{G}^{\circ}\left(\mathcal{M}_{R}\right), R=\mathbf{Z}$ or $\mathbf{Q}, n \geq 2$, and $\operatorname{proj} \operatorname{dim} \underline{M} \leq 1$. Then

(a) Moore $G$-spaces of type $(\underline{M}, n)$ exist and are unique, up to $G$-equivalence.

(b) Let $X$ be a Moore $G$-space of type $(\underline{M}, n)$, and let $Y$ be a $G$-space. Then there is a short-exact sequence of groups (abelian groups, if $n>2$ ),

$$
\operatorname{Ext}^{1}\left(\underline{M}, \underline{\pi}_{n+1} Y\right) \mapsto[X, Y] \rightarrow \operatorname{Hom}\left(\underline{M}, \underline{\pi}_{n} Y\right) .
$$

PROOF. The hypothesis proj $\operatorname{dim} \underline{M} \leq 1$ implies that $\underline{M}$ has a free resolution of the form

$$
\underline{F}\left[X_{1}\right] \stackrel{\partial_{1}}{\longmapsto} \underline{F}\left[X_{0}\right] \rightarrow \underline{M}
$$

(allowing the case $X_{1}=\varnothing$ ). Use 2.5 .2 to represent $\underline{\partial}_{1}$ by a $G$-map

$$
S^{n} \wedge X_{1}^{+} \stackrel{d_{1}}{\rightarrow} S^{n} \wedge X_{0}^{+} \text {. }
$$

The desired Moore $G$-space is just the mapping cone $\hat{c}\left(d_{1}\right)$. This proves the existence assertion of (a). Uniqueness will follow from the proof of (b).

To prove (b), first note that $d_{1}$ may be taken to be a suspension (in fact, a double suspension if $n>2$ ). To see this, consider the diagram

$$
\begin{array}{cc}
\underline{\partial}_{1}^{\prime}-\underline{\pi}_{n-1}\left(S^{n-1} \wedge X_{0}^{+}\right) \\
\downarrow \text { hur } \\
\Phi X_{1}^{-} \rightarrow \underline{F}\left[X_{1}\right] \stackrel{\underline{\partial}_{1}}{\rightarrow} \underline{F}\left[X_{0}\right]=\underline{\tilde{H}}_{n-1}\left(S^{n-1} \wedge X_{0}^{+}\right)
\end{array}
$$

regarded as a diagram of $O_{G}$-sets. The $O_{G}$-set $\Phi X_{1}$ is projective, and hur is always epi, so the lift $\partial_{1}^{\prime}$ exists. Using 2.5.2, choose $d_{1}^{\prime}: S^{n-1} \wedge X_{1}^{+} \rightarrow S^{n-1} \wedge X_{0}^{+}$to realize $\partial_{1}^{\prime}$, and set $d_{1}=\sum d_{1}^{\prime}$. A similar argument gets a double suspension when $n>2$.

It follows that $\hat{c}\left(d_{1}\right)$ may be taken to be a suspension (double suspension when $n>2$ ). Now consider the equivariant Puppe sequence [B, III.4] associated with $d_{1}$,

$$
S^{n} \wedge X_{1}^{+} \stackrel{d_{1}}{\rightarrow} S^{n} \wedge X_{0}^{+} \rightarrow \hat{c}\left(d_{1}\right) \rightarrow S^{n+1} \wedge X_{1}^{+} \stackrel{\sum d_{1}}{\rightarrow} \cdots
$$

and form the exact mapping-set sequence

$$
\left[S^{n} \wedge X_{1}^{+}, Y\right] \stackrel{d_{1}^{*}}{\leftarrow}\left[S^{n} \wedge X_{0}^{+}, Y\right] \leftarrow\left[\hat{c}\left(d_{1}\right), Y\right] \stackrel{\sum d_{1}^{*}}{\leftarrow} \cdots .
$$

Set $\underline{F}_{i}=\underline{F}\left[X_{i}\right]$. By 2.5.2, (1) becomes

(2)

$$
\begin{aligned}
\operatorname{Hom}\left(\underline{F}_{1}, \underline{\pi}_{n} Y\right) \stackrel{d_{i}^{*}}{\leftarrow} \operatorname{Hom}\left(\underline{F}_{0}, \underline{\pi}_{n} Y\right) \\
\left.\qquad \hat{c}\left(d_{1}\right), Y\right] \leftarrow \operatorname{Hom}\left(\underline{F}_{1}, \underline{\pi}_{n+1} Y\right) \stackrel{d_{i}^{*}}{\leftarrow} \operatorname{Hom}\left(\underline{F}_{0}, \underline{\pi}_{n+1} Y\right) .
\end{aligned}
$$

The usual considerations involving group structures apply here, and we see that (2) yields exact sequence of groups (abelian, if $n>2$ )

$$
\operatorname{Ext}^{1}\left(\underline{M}, \underline{\pi}_{n+1} Y\right) \longmapsto\left[\hat{c}\left(d_{1}\right), Y\right] \rightarrow \operatorname{Hom}\left(\underline{M}, \underline{\pi}_{n} Y\right) .
$$


Now choose $Y=X$, where $X$ is any Moore $G$-space of type $(\underline{M}, n)$. Then, we have $\underline{\pi}_{n} Y=\underline{\pi}_{n} X=\underline{\tilde{H}}_{n} X=\underline{M}$, and the above sequence shows that we may choose a $G$-map $\hat{c}\left(d_{1}\right) \rightarrow X$ inducing an equivalence $\underline{\tilde{H}}_{*} \hat{c}\left(d_{1}\right) \approx \underline{\tilde{H}}_{*} X$. Thus, $\hat{c}\left(d_{1}\right) \simeq X$, and we may substitute $X$ for $\hat{c}\left(d_{1}\right)$ in the above sequence, proving (b).

In fact, we have also verified the uniqueness assertion of (a), since we have just shown that all Moore $G$-spaces of type $(\underline{M}, n)$ are $G$-equivalent to $\hat{c}\left(d_{1}\right)$.

Recall (1.2) that a $G$-space $X$ is called a classical Moore $G$-space of type $(M, n)$, $M$ some $\mathbf{Z} G$-module, if $X$ is 1 -connected, $\tilde{H}_{n} X \approx M$, and $\tilde{H}_{i} X=0, i \neq n$. We shall use the relation of weak $G$-homotopy type for such spaces. This is generated by $G$-maps which are weak homotopy equivalences.

4.3. Corollary. Choose $n \geq 2$, let $G$ be any finite group, and $M$ any $\mathbf{Z} G$ module with proj $\operatorname{dim} M<\infty$. Then, classical Moore $G$-spaces of type $(M, n)$ exist and have unique weak $G$-homotopy type. Similarly when $M$ is any $\mathbf{Q} G$-module.

PROOF. By a well-known theorem of Rim, proj $\operatorname{dim} M \leq 1$. Existence now has a standard cell-attachment proof, first used by Swan [Sw]. It will, however, be useful to derive existence from 4.2. Accordingly, we define an $O_{G}$-module $\underline{M}$ by $\underline{M}(G / e)=M, \underline{M}(G / H)=0, H \neq e$. The association $M \mapsto \underline{M}$ may also be applied to the projective modules in a length-one resolution of $M$, yielding a length-one projective $O_{G}$-resolution of $\underline{M}$. Thus, proj $\operatorname{dim} \underline{M} \leq 1$. Any Moore $G$-space of type $(\underline{M}, n)$ is a classical Moore $G$-space of type $(M, n)$, so that existence now follows from 4.2 .

To prove uniqueness, start with a classical Moore $G$-space $X$ of type $(M, n)$, $n \geq 2$, proj $\operatorname{dim} M \leq 1$, and construct a $G$-map $e: Y \rightarrow X$ such that

(a) $e$ is a weak homotopy equivalence, and

(b) $Y$ is a Moore $G$-space of type $(\underline{M}, n)$, where $\underline{M}(G / e)=M$ and $\underline{M}(G / H)=0$, $H \neq e$.

In fact, $Y$ may be constructed by choosing a free, contractible $G$-space $E$ and adjoining a cone to $X \times E$ at ${ }^{*} \times E$. The map $e$ is just the trivial extension of the projection $X \times E \rightarrow X$.

As argued above, $\operatorname{proj} \operatorname{dim} \underline{M} \leq 1$, so we have shown that every classical Moore $G$-space of type $(M, n)$ has the weak $G$-homotopy type of a Moore $G$-space of type $(\underline{M}, n)$ with proj $\operatorname{dim} \underline{M} \leq 1$. Since, by 4.2 , any two of the latter are $G$-equivalent, we have verified uniqueness.

We next obtain a result which is a slight diversion from our main interest but which will help in the formulation of our last two theorems. Recall that the free dimension of an $O_{G}$-module $\underline{M}$, written fr $\operatorname{dim} \underline{M}$, is the minimum length of all free $O_{G}$-resolutions of $\underline{M}$. It deviates from proj $\operatorname{dim} \underline{M}$ only when $\underline{M}$ is projective but not free. We also refer the reader to the comments about hodim above 3.2.

4.4. Corollary. Let $K \in G-R \mathcal{W}_{*}^{1}$ be a Moore $G$-space of type $(\underline{M}, n), \underline{M} \neq$ 0. Then

(a) if $n=2$, hodim $K=n+\operatorname{fr} \operatorname{dim} \underline{M}$;

(b) if $n>2$, hodim $K=n+\operatorname{proj} \operatorname{dim} \underline{M}$.

ProOF. Let $L_{1}, L_{2} \in G-R W_{*}^{1}$ be $G$-spaces, $G$-equivalent to $K$, with $L_{2}$ realizing a minimal, free degree $n$ resolution of $\underline{M}$. Note that $\underline{\tilde{C}}_{*} L_{1}=\underline{A} \oplus \underline{B}$, with $\underline{A}$ acyclic 
and at most $n$-dimensional and $\underline{B}$ a projective, degree $n$ resolution of $\underline{M}$. Thus,

$$
n+\operatorname{proj} \operatorname{dim} \underline{M} \leq \operatorname{dim} L_{1} \text {. }
$$

Since this holds for any $L_{1} \simeq K, L_{1} \in G$-R $\mathcal{W}_{*}^{1}$, we have

$$
n+\operatorname{proj} \operatorname{dim} \underline{M} \leq \operatorname{hodim} K .
$$

On the other hand, by construction,

$$
n+\operatorname{fr} \operatorname{dim} \underline{M}=\operatorname{dim} L_{2} \geq \operatorname{hodim} L_{2}=\operatorname{hodim} K,
$$

so that if $\operatorname{proj} \operatorname{dim} \underline{M}>0$ or $\underline{M}$ is free, then the inequalities all reduce to equalities and we are done.

Henceforth assume that $\underline{M}$ is projective but not free.

Suppose $n=2$. Since $G$-complexes, here, are always restricted to $G-R W_{*}^{1}$, a 2 dimensional $G$-complex is a bouquet of 2 -spheres and, hence, has a free $\underline{\tilde{H}}_{2}$. Thus, hodim $K=3=2+\operatorname{fr} \operatorname{dim} \underline{M}$, as required.

When $n>2$, we use the Eilenberg trick to find a short, split-exact sequence $\underline{M} \mapsto \underline{F}_{0} \stackrel{\alpha}{\rightarrow} F_{1}$, with $\underline{F}_{i}=\underline{F}\left[X_{i}\right]$ free. Realize $\underline{\alpha}$ by a $G$-map $a: S^{n-1} \wedge X_{0}^{+} \rightarrow$ $S^{n-1} \wedge X_{1}^{+}$(2.5.2), and let $L$ be the mapping cone $\hat{c}(a)$. Clearly, $L$ has dimension $n$ and is a Moore $G$-space of type $(\underline{M}, n)$. By the uniqueness assertion in 4.2(a), $L \simeq K$, so that hodim $K=n$, as required.

For the next result, we recall the stable mapping sets

$$
\left\{K_{1}, K_{2}\right\}=\operatorname{colim}\left[\sum^{l} K_{1}, \sum^{l} K_{2}\right]
$$

and the derived functors $\mathrm{Ext}^{i}$ of Hom. It will be convenient to set $\mathrm{Ext}^{i}=0$ for $i<0$.

4.5. THEOREM. Suppose that $\underline{M}_{i} \in \mathcal{O}_{G}^{\circ}\left(\mathcal{M}_{\mathbf{Q}}\right), n_{i} \geq 2$, and that $K_{i}$ is a Moore $G$-space of type $\left(M_{i}, n_{i}\right), i=1,2$. There is a natural isomorphism of abelian groups $\left\{K_{1}, K_{2}\right\} \approx \operatorname{Ext}^{n_{2}-n_{1}}\left(\underline{M}_{1}, \underline{M}_{2}\right)$. When $n_{1}=n_{2}=n$, this is just the map induced by $\underline{\tilde{H}}_{n}$. The natural map $\left[K_{1}, K_{2}\right] \rightarrow\left\{K_{1}, K_{2}\right\}$ is

(a) surjective, if $2 n_{2}-n_{1} \geq \operatorname{proj} \operatorname{dim} \underline{M}_{1}+1$,

(b) bijective, if $2 n_{2}-n_{1} \geq \operatorname{proj} \operatorname{dim} \underline{M}_{1}+2$.

PROOF. We want to apply Corollary 3.9 , which requires verifying that hodim $K_{1}$ $<\infty$. By 4.4 , this is equivalent to checking that proj $\operatorname{dim} \underline{M}_{1}<\infty$, which holds by the lemma in 2.2.2.

To apply 3.9 , note that $\underline{\tilde{H}}_{q} K_{2}=0$ unless $q=n_{2}$. Now use 3.9 (b) to get $E_{p q}^{\infty}=E_{p q}^{2}=0$, for $q \neq n_{2}$, and $E_{p n_{2}}^{\infty}=\operatorname{Ext}^{-p-n_{1}}\left(\underline{M}_{1}, \underline{M}_{2}\right)$. Thus, the filtration of $\left\{K_{1}, K_{2}\right\}$ consists only of $E_{-n_{2} n_{2}}^{2}=\operatorname{Ext}^{n_{2}-n_{1}}\left(\underline{M}_{1}, \underline{M}_{2}\right)$, as asserted.

To verify (a) and (b), we first assume $n_{1}>2$ or proj $\operatorname{dim} \underline{M}_{1} \geq 1$. In either case, by 4.4 , hodim $K_{1}=n_{1}+\operatorname{proj} \operatorname{dim} \underline{M}_{1}$. Also, conn $K_{2}=n_{2}-1$. Thus, the inequalities in (a) and (b) are equivalent to

$\left(\mathrm{a}^{\prime}\right) \operatorname{hodim} K_{1} \leq 2$ conn $K_{2}+1$,

(b') hodim $K_{1} \leq 2 \underline{\text { conn }} K_{2}$,

respectively, and these are precisely the conditions in 3.8 that imply the desired surjectivity and bijectivity, respectively. 
When $n_{1}=2$ and $\operatorname{proj} \operatorname{dim} \underline{M}_{1}=0$, we apply Theorem 4.2 to get $\left[K_{1}, K_{2}\right] \approx$ $\operatorname{Hom}\left(\underline{M}_{1}, \underline{\pi}_{2} \underline{K}_{2}\right)$. This equals $\operatorname{Hom}\left(\underline{M}_{1}, \underline{M}_{2}\right)$ or 0 according as $n_{2}=2$ or $n_{2}>2$. But so does $\operatorname{Ext}^{n_{2}-2}\left(\underline{M}_{1}, \underline{M}_{2}\right) \approx\left\{\underline{K}_{1}, \underline{K}_{2}\right\}$, and it is not hard to see that these identifications correspond to the natural map $\left[K_{1}, K_{2}\right] \rightarrow\left\{K_{1}, K_{2}\right\}$. Thus (a) and (b) hold in this case as well.

Finally, we conclude with our uniqueness result (Theorem A of $\S 1$ ).

4.6. CoRollary. Choose $\underline{M} \in \mathrm{O}_{G}^{\circ}\left(\mathcal{M}_{\mathbf{Q}}\right)$. All Moore $G$-complexes realizing $\underline{M}$ are stably $G$-equivalent. In fact, if

$$
n \geq \operatorname{proj} \operatorname{dim} \underline{M}+1,
$$

then all Moore $G$-spaces of type $(\underline{M}, n)$ are $G$-equivalent.

PROOF. It suffices to prove the second assertion. If both $K_{1}$ and $K_{2}$ realize $(\underline{M}, n)$, with $n$ as above, then we can apply $4.5\left(\right.$ a) to get a $G$-map $K_{1} \rightarrow K_{2}$ which is a homology equivalence, hence a $G$-equivalence.

5. Two nonequivalent Moore $G$-spaces of type $(\underline{M}, 2)$. Throughout this section, we shall be working with rational $G$-spaces. Thus, as mentioned in $1.3, S^{n}$ will denote the rational $n$-sphere, $n \geq 1$. Correspondingly, we restrict entirely to $O_{G}$-modules in $O_{G}^{\circ}\left(\mathcal{M}_{\mathbf{Q}}\right)$ and $O_{G}$-chain-complexes in $C h^{1}\left(O_{G}^{\circ}\left(\mathcal{M}_{\mathbf{Q}}\right)\right)$.

5.1. THEOREM. Let $G=\mathbf{Z} / 2 \oplus \mathbf{Z} / 2$. There exists an $\underline{M}$ in $O_{G}^{\circ}\left(\mathcal{M}_{\mathbf{Q}}\right)$ and two Moore $G$-spaces $L_{1}$ and $L_{2}$ of type $(\underline{M}, 2)$ that are not $G$-equivalent.

By the lemma in 2.2.2, every $O_{G}$-module has projective dimension $\leq 2$ in this case, and so Corollary 4.6 implies that $\sum L_{1} \simeq \sum L_{2}$.

The proof of 5.1 is long, occupying the rest of this section. To aid the reader, we begin with an outline of the construction.

5.2. Outline of the construction.

Step 1 . Choose an $O_{G}$-module $\underline{M}$ such that

$$
\operatorname{Hom}(\underline{M}, \underline{M})=\mathbf{Q}^{2} \text { and } \operatorname{Ext}^{2}(\underline{M}, \underline{M}) \neq 0 \text {. }
$$

Such an $\underline{M}$ has projective dimension 2 , so we may choose a free resolution

$$
\underline{M} \stackrel{\varepsilon}{\leftarrow} \underline{F}\left[X_{0}\right] \stackrel{\underline{\partial}_{1}}{\leftarrow} \underline{F}\left[X_{1}\right] \stackrel{\underline{\partial}_{2}}{\leftarrow} \underline{F}\left[X_{2}\right] .
$$

Step 2. Begin building a $G$-complex that realizes the resolution, as in the proof of Theorem 3.3.

Choose $d_{1}: S^{2} \wedge X_{1}^{+} \rightarrow S^{2} \wedge X_{0}^{+}$to induce $\underline{\partial}_{1}$ on $\underline{H}_{2}=\underline{\pi}_{2}$. By $2.5 .2, d_{1}$ is unique, up to $G$-homotopy. Set $K$ equal to the mapping cone $\hat{c}\left(d_{1}\right)$, and choose $d_{2}: S^{3} \wedge X_{2}^{+} \rightarrow K$ to induce $\underline{\partial}_{2}: \underline{F}\left[X_{2}\right] \rightarrow \operatorname{ker} \underline{\partial}_{1}=\underline{H}_{3} K$. There are many such choices. Set $L_{1}=\hat{c}\left(d_{2}\right)$.

5.2.1. PROPOSITION. There exists a choice of $d_{2}$ such that the natural map $\left[L_{1}, L_{1}\right] \rightarrow \operatorname{Hom}(\underline{M}, \underline{M})$ is surjective.

This will be proved in Step 5. Henceforth, fix $d_{2}$ as in 5.2.1.

Step 3. Define a homomorphism

$$
\Delta: \operatorname{Hom}\left(\underline{F}\left[X_{2}\right], \underline{\pi}_{3} K\right) \rightarrow \operatorname{Ext}^{2}(\underline{M}, \underline{M})
$$


which, roughly, measures the difference between $d_{2}$ and other candidates for attaching maps $S^{3} \wedge X_{2}^{+} \rightarrow K$.

Choose $\tilde{d}_{2}: S^{3} \wedge X_{2}^{+} \rightarrow K$ so as to induce $\underline{\partial}_{2}$ and so that $\Delta\left(\tilde{d}_{2}\right) \neq 0$. Set $L_{2}=\hat{c}\left(\tilde{d}_{2}\right)$.

Step 4. Prove that $\Delta\left(\tilde{d}_{2}\right) \neq 0$ implies that there can be no $G$-map $L_{1} \rightarrow L_{2}$ inducing the identity $\underline{M}=\underline{H}_{2} L_{1} \rightarrow \underline{H}_{2} L_{2}=\underline{M}$.

Using 5.2.1, it follows that no $G$-map $L_{1} \rightarrow L_{2}$ induces an automorphism $\underline{M} \rightarrow$ $\underline{M}$, and thus $L_{1} \not L_{2}$.

Step 5. Prove Proposition 5.2.1.

Here we actually prove two propositions $\left(5.4 .4^{\prime}\right.$ and $\left.5.4 .4^{\prime \prime}\right)$ that imply 5.2.1. The idea is that $L_{1}$ is composed of two pieces, and we find it convenient to prove a 5.2.1-like result for each. The proofs use an equivariant Hopf invariant that enables us to suitably modify a first-approximation.

5.3. Step 1. The choice of $\underline{M}$. We first define some convenient notation. Useful references are [T1 and T3]. Recall that $G=\mathbf{Z} / \mathbf{2} \oplus \mathbf{Z} / \mathbf{2}$.

$G$ contains five subgroups, $G, e, H_{1}, H_{2}, H_{3}$ which we find convenient to arrange as follows.

$$
G \begin{array}{ll}
H_{1} & \\
H_{2} & e \\
H_{3} &
\end{array}
$$

Inclusions can be thought of as proceeding from right to left, and these correspond to projections of corresponding coset spaces in $O_{G}$. An $O_{G}$-module $\underline{N}$ may then be arranged as follows.

$$
\underline{N} \quad \quad \quad \begin{array}{lll}
N_{1} & \\
& N_{2} & M \\
& N_{3} &
\end{array}
$$

Here $\underline{N}(G / G)=V$ is a $\mathbf{Q}$-vector space, $\underline{N}\left(G / H_{i}\right)=N_{i}$ is a $\mathbf{Q}\left[G / H_{i}\right]$-module, and $\underline{N}(G / e)=M$ is a $\mathbf{Q}[G]$-module. Suitable module homomorphisms $\underline{N}(G / H) \rightarrow$ $\underline{N}(G / K)$ exist, corresponding to projections $G / K \rightarrow G / H$ (equivalently, inclusions $K \leq H)$, and these proceed from left to right. We shall not need to display these explicitly in our notation.

We always give the rationals $\mathbf{Q}$ the trivial $\mathbf{Q}[G / H]$-module structure.

We begin by listing examples of projective $O_{G}$-modules. All the module homomorphisms are standard inclusions (see $[\mathbf{T 1}, \S 3]$ ):

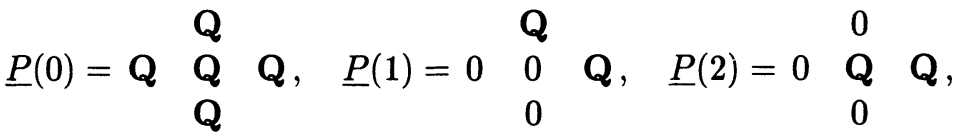

$$
\begin{aligned}
& \underline{P}(3)=\begin{array}{lll} 
& 0 \\
0 & 0 \\
& \mathbf{Q}
\end{array} \quad \mathbf{Q}, \quad \underline{P}(4)=\begin{array}{lll}
0 & 0 & 0 \\
& & 0
\end{array} .
\end{aligned}
$$

In fact, note that $\underline{P}(0)$ is the free $O_{G}$-module $\underline{F}[G / G]$, where $G / G$ is the singleton $G$-set. 
To define $\underline{M}$, we describe one more auxiliary $\mathcal{O}_{G}$-module,

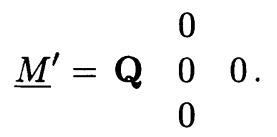

It is easy to verify directly that $\underline{M}^{\prime}$ is injective. Now set

$$
\underline{M}=\underline{M}^{\prime}+\underline{P}(4)=\mathbf{Q} \begin{array}{ll}
0 \\
0 \\
0
\end{array} \quad \mathbf{Q} .
$$

Note that

$$
\operatorname{Ext}^{i}(\underline{M}, \underline{M})= \begin{cases}\operatorname{Hom}(\underline{M}, \underline{M})=\mathbf{Q}^{2}, & i=0, \\ \operatorname{Ext}^{i}\left(\underline{M}^{\prime}, \underline{P}(4)\right), & i>0 .\end{cases}
$$

To compute $\operatorname{Ext}^{i}\left(\underline{M}^{\prime}, \underline{P}(4)\right)$, we construct a minimal projective resolution for $\underline{M}^{\prime}$, using the projective-cover construction of $[\mathbf{T} \mathbf{1}]$ : all maps are obvious inclusions or projections

$$
\begin{array}{cccc}
\underline{P}_{0} & \underline{P}_{1} & \underline{P}_{2} \\
\underline{M}^{\prime} \leftarrow \quad \leftarrow(0) & \leftarrow \quad \underline{P}(1)+\underline{P}(2)+\underline{P}(3) & \leftarrow & \underline{P}(4)+\underline{P}(4) .
\end{array}
$$

Apply $\operatorname{Hom}(-, P(4))$ to this resolution. One verifies easily that the resulting complex is $0 \rightarrow 0 \rightarrow \mathbf{Q}^{2}$. Therefore,

$$
\operatorname{Ext}^{i}(\underline{M}, \underline{M})= \begin{cases}\mathbf{Q}^{2}, & i=0 \text { or } 2, \\ 0, & \text { otherwise. }\end{cases}
$$

Now we convert (5.3.1) to a free resolution of $\underline{M}^{\prime}$ by selecting a free module $\underline{F}^{\prime}$ such that $\underline{P}_{i}+\underline{F}^{\prime}=\underline{F}_{i}^{\prime}$ is free, $i=1,2$. This uses Eilenberg's trick. Set $\underline{F}_{0}^{\prime}=\underline{P}(\overline{0})$. Then, we get

$$
\underline{R}^{\prime}: \underline{M}^{\prime} \nleftarrow \underline{F}_{0}^{\prime} \stackrel{\underline{\partial}_{1}^{\prime}}{\leftarrow} \underline{F}_{1}^{\prime} \stackrel{\underline{\partial}_{2}^{\prime \prime}}{\leftrightarrow} \underline{F}_{2}^{\prime} .
$$

Next we choose the free $G$-set $G$ and corresponding free $O_{G}$-module $\underline{F}_{0}^{\prime \prime}=\underline{F}[G]$ (cf. 2.1). It satisfies $\underline{F}_{0}^{\prime \prime}(G / H)=0$, for $H \neq e$, and $\underline{F}_{0}^{\prime \prime}(G / e)=\mathbf{Q}[G]$. Thus, the standard augmentation $\eta: \mathbf{Q}[G] \rightarrow \mathbf{Q}$ gives an epimorphism $\underline{F}_{0}^{\prime \prime} \rightarrow \underline{P}(4)$ with (projective) kernel $\underline{N}$. Again use Eilenberg's trick to find a free $\underline{F}_{2}^{\prime \prime}$ with $\underline{F}_{1}^{\prime \prime}=$ $\underline{N}+\underline{F}_{2}^{\prime \prime}$ free. We set $\underline{M}^{\prime \prime}=\underline{P}(4)$ and obtain a free resolution

$$
\underline{R}^{\prime \prime}: \underline{M}^{\prime \prime} \leftarrow \underline{F}_{0}^{\prime \prime} \stackrel{\partial_{1}^{\prime \prime}}{\leftarrow} \underline{F}_{1}^{\prime \prime} \stackrel{\underline{\partial}_{2}^{\prime \prime}}{\leftarrow} \underline{F}_{2}^{\prime \prime} .
$$

Let $\underline{F}_{i}^{\prime}=\underline{F}\left[X_{i}^{\prime}\right], \underline{F}_{i}^{\prime \prime}=\underline{F}\left[X_{i}^{\prime \prime}\right]$, and $\underline{F}_{i}=\underline{F}\left[X_{i}\right]$, with $X_{i}=X_{i}^{\prime} \cup X_{i}^{\prime \prime}, i=0,1,2$. We thus have a free resolution

$$
\underline{R}: \underline{M} \nleftarrow \underline{F}_{0} \stackrel{\underline{\partial}_{0}}{\leftarrow} \underline{F}_{1} \stackrel{\underline{\partial}_{1}}{\leftarrow} \underline{F}_{2},
$$

with $\underline{R}=\underline{R}^{\prime}+\underline{R}^{\prime \prime}$.

5.4. Step 2. The construction of $L_{1}$ realizing (5.3.5). We shall realize $\sum^{2} \underline{R}^{\prime}$ and $\sum^{2} \underline{R}^{\prime \prime}$ by $G$-complexes $L_{1}^{\prime}$ and $L_{1}^{\prime \prime}$, respectively, and then we set $L_{1}=L_{1}^{\prime} \vee L_{1}^{\prime \prime}$, which, of course, realizes $\sum^{2} R$. These will all be 4 -dimensional. 
In the rest of this step, we make a number of remarks about $L_{1}^{\prime}$ and $\underline{R^{\prime}}$. These may all be converted to equally valid assertions about $L_{1}$ and $\underline{R}$ or $L_{1}^{\prime \prime}$ and $\underline{R}^{\prime \prime}$ by decreasing or increasing the number of superscript primes in the notation.

Since $\sum^{2} \underline{R}^{\prime}$ satisfies the condition hodim $\leq 2$ conn +2 of Theorem 3.3 , we may take $L_{1}^{\prime}$ to be the realization produced by the proof of 3.3 . Let $d_{i}^{\prime}$ denote the attaching map in the construction of $L_{1}^{\prime}$ that corresponds to the boundary map $\underline{\partial}_{i}^{\prime}$ in $\underline{R}^{\prime}$. For reference, note that the domain of $d_{i}^{\prime}$ is the bouquet $S^{i+1} \wedge X_{i}^{\prime+}, i=1,2$. Let $K^{\prime}$ be the mapping cone $\hat{c}\left(d_{1}^{\prime}\right)$, the 3-skeleton of $L_{1}^{\prime}$.

Just as in the proof of 4.2 , using 2.5 .2 , we may choose $d_{1}^{\prime}$ to be a suspension. Hence $K^{\prime}$ is a suspension. We have

$$
\underline{H}_{3} K^{\prime}=\operatorname{ker} \underline{\partial}_{1}^{\prime}=\operatorname{im} \underline{\partial}_{2}^{\prime}=\underline{F}_{2}^{\prime} .
$$

We shall make frequent use of these identifications, as well as the abbreviations

$$
\underline{H}_{3}^{\prime}=\underline{H}_{3} K^{\prime}, \quad \underline{\pi}_{3}^{\prime}=\underline{\pi}_{3} K^{\prime} .
$$

Note that $d_{2}^{\prime}: S^{3} \wedge X_{2}^{\prime+} \rightarrow K^{\prime}$ determines a commutative diagram

$$
\begin{array}{ccc}
\underline{\pi}_{3} S^{3} \wedge X_{2}^{\prime+} & \stackrel{d_{2 *}^{\prime}}{\rightarrow} & \underline{\pi}_{3}^{\prime} \\
\text { hur } \downarrow \approx & & \downarrow \text { hur } \\
\underline{H}_{3} S^{3} \wedge X_{2}^{\prime+} & \stackrel{\underline{\partial}_{2}^{\prime}}{\longrightarrow} & \underline{H}_{3}^{\prime} \\
\| & & \| \\
\underline{F}_{2}^{\prime} & \stackrel{\underline{\partial}_{2}^{\prime}}{\approx} & \operatorname{ker} \underline{\partial}_{1}^{\prime}
\end{array}
$$

We may describe this by saying that $d_{2}^{\prime}$ lifts $\underline{\partial}_{2}^{\prime}$ or, using (5.4.3) to identify $\underline{\pi}_{3} S^{3} \wedge$ $X_{2}^{\prime+}$ with $\underline{H}_{3}^{\prime}$, we may say $d_{2}^{\prime}$ splits hur.

Now $d_{2}^{\prime}$ is not uniquely determined by the construction in the proof of 3.3 (even up to $G$-homotopy).

5.4.4'. Proposition. $d_{2}^{\prime}$ may be chosen so that $\left[L_{1}^{\prime}, L_{1}^{\prime}\right] \rightarrow \operatorname{Hom}\left(M^{\prime}, M^{\prime}\right)$ is surjective.

Since $\operatorname{Hom}(\underline{M}, \underline{M})=\operatorname{Hom}\left(\underline{M}^{\prime}, \underline{M}^{\prime}\right)+\operatorname{Hom}\left(M^{\prime \prime}, M^{\prime \prime}\right)$, it is easy to see that 5.4.4' and (5.4.4") imply Proposition 5.2.1, i.e.,

$$
\left[L_{1}, L_{1}\right] \rightarrow \operatorname{Hom}(\underline{M}, \underline{M}) \text { is surjective. }
$$

We prove 5.4.4' and (5.4.4") in Step 5. Henceforth, we assume 5.4.4. We do not need to refer further to $L_{1}^{\prime}, L_{1}^{\prime \prime}$ except in Step 5 .

5.5. Step 3. The homomorphism $\Delta: \operatorname{Hom}\left(\underline{H}_{3}, \underline{\pi}_{3}\right) \rightarrow \operatorname{Ext}^{2}(\underline{M}, \underline{M})$. Note first that

$$
\underline{\pi}_{3} L_{1}=\underline{M} .
$$

This is immediate from the choice of

$$
\underline{M}=\mathbf{Q} \begin{array}{ll}
0 \\
0 \\
0
\end{array} \quad \mathbf{Q}
$$


and the construction of $L_{1}$, since

$$
L_{1}^{H} \simeq \begin{cases}S^{2}, & H=G, e \\ *, & \text { otherwise }\end{cases}
$$

Next, using (5.4.1) (unprimed), we identify $\underline{\partial}_{2}: \underline{F}_{2} \rightarrow \underline{F}_{1}$ with the inclusion $\underline{H}_{3} \mapsto \underline{F}_{1}$. By a standard property of Ext*, we have, for any $O_{G}$-module $\underline{B}$, an exact sequence

$$
\operatorname{Hom}\left(\underline{F}_{1}, \underline{B}\right) \rightarrow \operatorname{Hom}\left(\underline{H}_{3}, \underline{B}\right) \rightarrow \operatorname{Ext}^{2}(\underline{M}, \underline{B}) .
$$

We are particularly interested in the case $\underline{B}=\underline{\pi}_{3}$.

Observe that we have a commutative diagram

$$
\begin{aligned}
& \underline{\pi}_{4}\left(L_{1}, K\right) \rightarrow \underline{\pi}_{3} \rightarrow \underline{\pi}_{3} L_{1}=\underline{M} \\
& \quad \chi \quad \downarrow \text { hur } \\
& \underline{\pi}_{3}\left(S^{3} \wedge X_{2}^{+}\right) \approx \underline{H}_{3}
\end{aligned}
$$

so that, essentially, $d_{2}$ gives a splitting $\underline{\pi}_{3}=\underline{H}_{3} \oplus \underline{M}$.

Plugging this value of $\underline{B}$ into the second and third terms in (5.5.2) yields compatible splittings

$$
\begin{gathered}
\operatorname{Hom}\left(\underline{H}_{3}, \underline{\pi}_{3}\right)=\operatorname{Hom}\left(\underline{H}_{3}, \underline{H}_{3}\right) \oplus \operatorname{Hom}\left(\underline{H}_{3}, \underline{M}\right) \\
\downarrow \\
\operatorname{Ext}^{2}\left(\underline{M}, \underline{\pi}_{3}\right)=\operatorname{Ext}^{2}\left(\underline{M}, \underline{H}_{3}\right) \oplus \operatorname{Ext}^{2}(\underline{M}, \underline{M})
\end{gathered}
$$

The homomorphism $\Delta$ is now defined to be the composite

$$
\operatorname{Hom}\left(\underline{H}_{3}, \underline{\pi}_{3}\right) \rightarrow \operatorname{Ext}^{2}\left(\underline{M}, \underline{H}_{3}\right) \oplus \operatorname{Ext}^{2}(\underline{M}, \underline{M}) \stackrel{\mathrm{pr}}{\rightarrow} \operatorname{Ext}^{2}(\underline{M}, \underline{M}) \text {. }
$$

Note that since $d_{2}$ projects to 0 in $\operatorname{Hom}\left(\underline{H}_{3}, \underline{M}\right)$,

$$
\Delta\left(d_{2}\right)=0 \text {. }
$$

Choose $\tilde{d}_{2}: S^{3} \wedge X_{2}^{+} \rightarrow K$ as follows. By abuse of notation, we have $\tilde{d}_{2} \in$ $\operatorname{Hom}\left(\underline{H}_{3}, \underline{\pi}_{3}\right)$. We require that $\tilde{d}_{2}$ project onto id $\underline{H}_{3}$ in $\operatorname{Hom}\left(\underline{H}_{3}, \underline{H}_{3}\right)$, i.e., $\tilde{d}_{2}$ lifts $\underline{\partial}_{2}$, and

$$
\Delta\left(\tilde{d}_{2}\right) \neq 0 .
$$

Since $\operatorname{Hom}\left(\underline{H}_{3}, \underline{M}\right) \rightarrow \operatorname{Ext}^{2}(\underline{M}, \underline{M})=\mathbf{Q}^{2}$ is surjective, such a choice is possible.

Fix such a $\tilde{d}_{2}$ and set $L_{2}=\hat{c}\left(\tilde{d}_{2}\right)$.

5.6. Step 4. Self-maps $K \rightarrow K$ and maps $L_{1} \rightarrow L_{2}$. We shall make use of the operation of $\left[S^{3} \wedge X_{1}^{+}, K\right]$ on $[K, K]$, which is defined just as in the nonequivariant case. That is, if $p: K \rightarrow K \vee\left(S^{3} \wedge X_{1}^{+}\right)$is the pinching map-recall that $K$ is the mapping cone of $d_{1}: S^{2} \wedge X_{1}^{+} \rightarrow S^{2} \wedge X_{0}^{+}$-and $a: S^{3} \wedge X_{1}^{+} \rightarrow K, f: K \rightarrow K$ are arbitrary $G$-maps, then $f^{a}$ is given by the composite

$$
K \underset{p}{\rightarrow} K \vee\left(S^{3} \wedge X_{1}^{+}\right) \underset{\{f, a\}}{\rightarrow} K .
$$

We shall abuse notation and use the same letters for $G$-homotopy classes.

Note that, by 2.5 .2 we have an identification

$$
\left[S^{3} \wedge X_{1}^{+}, K\right]=\operatorname{Hom}\left(\underline{F}_{1}, \underline{\pi}_{3}\right)
$$


which we use without further comment. The following commutative diagram introduces notation.

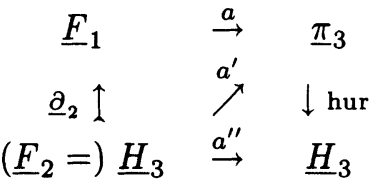

Our interest in the operation of $\operatorname{Hom}\left(\underline{F}_{1}, \underline{\pi}_{3}\right)$ on $[K, K]$ is explained by assertion (a) in the following lemma.

5.6.2. Lemma. (a) Let $1 \in[K, K]$ denote the class of the identity map. The orbit of 1 under the action of $\operatorname{Hom}\left(\underline{F}_{1}, \underline{\pi}_{3}\right)$ consists of all $G$-homotopy classes of $G$-maps $K \rightarrow K$ inducing id $\underline{M}_{\underline{M}}$ on $\underline{H}_{2}\left(=\underline{\pi}_{2}\right)$.

(b) The $\underline{\pi}_{3}$-endomorphism induced by $1^{a}$ is

$$
\mathrm{id}_{\underline{\pi}_{3}}+a^{\prime} \circ \text { hur. }
$$

(c) The $\underline{H}_{3}$-endomorphism induced by $1^{a}$ is

$$
\operatorname{id}_{\underline{H}_{3}}+a^{\prime \prime} \text {. }
$$

The proofs of (a)-(c) are the same as in the nonequivariant case and will be omitted.

5.6.3. LEMMA. Suppose that $f: K \rightarrow K$ is a $G$-map inducing the identity on $\underline{H}_{2} K=\underline{M}$. Then, there is no $G$-map $g: L_{1} \rightarrow L_{2}$ extending $f$.

PrOOF. We suppose $g$ exists and derive a contradiction.

Consider the commutative diagrams

(i)

$$
\begin{array}{lcc}
\underline{\pi}_{4}\left(D^{4} \wedge X_{2}^{+}, S^{3} \wedge X_{2}^{+}\right) & \underset{\approx}{\rightarrow} \underline{\pi}_{4}\left(L_{i}, K\right) \\
\quad \approx \downarrow & \downarrow \\
\underline{\pi}_{3}\left(S^{3} \wedge X_{2}^{+}\right) & \stackrel{d(i) *}{\longrightarrow} & \underline{\pi}_{3} K
\end{array}
$$

for $i=1,2$, where we have $d(1)=d_{2}, d(2)=\tilde{d}_{2}$. The map $g:\left(L_{1}, K\right) \rightarrow\left(L_{2}, K\right)$ induces a map of diagram (1) to diagram (2) and, thus a $G$-homotopy-commutative diagram

$$
\begin{array}{ccc}
S^{3} \wedge X_{2}^{+} & \stackrel{g^{\prime}}{\rightarrow} & S^{3} \wedge X_{2}^{+} \\
d_{2} \downarrow & & \downarrow \tilde{d}_{2} \\
K & \stackrel{f}{\rightarrow} & K
\end{array}
$$

We show that, in fact, the existence of (3) leads to a contradiction.

Note that, by $5.6 .2(\mathrm{a}), f=1^{a}$, for some $a \in \operatorname{Hom}\left(\underline{F}_{1}, \underline{\pi}_{3}\right)$, and, by $5.6 .2(\mathrm{~b})$,

$$
f \circ d_{2}-d_{2}=a^{\prime}
$$

as homomorphisms $\underline{H}_{3}=\underline{F}_{2}=\underline{\pi}_{3} S^{3} \wedge X_{2}^{+} \rightarrow \underline{\pi}_{3} K=\underline{\pi}_{3}$ (cf. 5.6.1). ${ }^{3}$

If we apply the functor $\underline{H}_{3}$ to (3), we see that the vertical maps reduce to the identification $\underline{F}_{2}=\underline{H}_{3} K=\underline{H}_{3}$. Thus, we may identify $\underline{H}_{3} g^{\prime}$ with $\underline{H}_{3} f$. In fact,

\footnotetext{
${ }^{3}$ We shall identify maps with their homotopy classes and the latter with induced homomorphisms, when appropriate, to simplify notation.
} 
since $\underline{\pi}_{3} S^{3} \wedge X_{2}^{+}=\underline{H}_{3} S^{3} \wedge X_{2}^{+}$, we may identify $\underline{\pi}_{3} g^{\prime}$ with $\underline{H}_{3} f$. Writing $g^{\prime}$ instead of $\underline{\pi}_{3} g^{\prime}$, and bearing in mind the identification $\underline{F}_{2}=\underline{H}_{3}$, we have $g^{\prime}=\operatorname{id}_{\underline{H}_{3}}+a^{\prime \prime}$, by $5.6 .2(\mathrm{c})$. Thus,

$$
\tilde{d}_{2} \circ g^{\prime}-\tilde{d}_{2}=\tilde{d}_{2} \circ a^{\prime \prime} .
$$

Now, by inspecting the right-hand sides of (4) and (5), we see that both of these homomorphisms $\underline{H}_{3} \rightarrow \underline{\pi}_{3}$ factor through (i.e., extend to) $\underline{F}_{1}$ (cf. 5.6.1). In view of the exact sequence (5.5.2), this implies that

$$
\Delta\left(f d_{2}-d_{2}\right)=0=\Delta\left(\tilde{d}_{2} g^{\prime}-\tilde{d}_{2}\right) .
$$

But the $G$-homotopy-commutativity of (3) gives

$$
\Delta\left(f d_{2}\right)=\Delta\left(\tilde{d}_{2} g^{\prime}\right),
$$

whereas our construction of $\Delta$ and $\tilde{d}_{2}$ in 5.5 implies that

$$
\Delta\left(d_{2}\right)=0 \neq \Delta\left(\tilde{d}_{2}\right) .
$$

Relations (6), (7), and (8) cannot hold simultaneously.

5.7. Step 5. Proof of Proposition 5.2.1. By the construction and discussion in 5.4, it suffices to prove (5.4.4') and $\left(5.4 .4^{\prime \prime}\right)$. Since both proofs are virtually the same, we adopt the following convention to simplify notation. We eliminate superscript primes and give both proofs simultaneously, with the exception of one computation (5.7.4(d)) which will be made separately for each case. Note, then, by our convention, in this section $\underline{M}$ will refer either to $\underline{M}^{\prime}$ or $\underline{M}^{\prime \prime}$ and not to the $\mathrm{O}_{G}$-module $\underline{M}^{\prime}+\underline{M}^{\prime \prime}$ of that name in Theorem 5.1 (cf. 5.3 for the definition of $\left.\underline{M}^{\prime}, \underline{M^{\prime \prime}}\right)$.

To begin, note that, in either case,

$$
\operatorname{Hom}(\underline{M}, \underline{M})=\mathbf{Q},
$$

where $r \in \mathbf{Q}$ corresponds to multiplication by $r$.

Let $S^{1}$ denote the rational circle, and let $f_{r}: S^{1} \rightarrow S^{1}$ denote a fixed map of degree $r$. Recall that the 3 -skeleton $K$ of $L_{1}$ is a suspension, say $K=\sum \bar{K}$. Thus, we have a $G$-equivalence $K=\sum \bar{K} \simeq S^{1} \wedge \bar{K}$. Let $e_{r}: K \rightarrow K$ denote the self- $G$ map corresponding to $f_{r} \wedge \mathrm{id}_{\bar{K}}$ under this $G$-equivalence. Proposition 5.4.4' and (5.4.4") will follow easily from

5.7.1. LEMMA. $d_{2}: S^{3} \wedge X_{2}^{+} \rightarrow K$ may be chosen so that

$$
\begin{array}{ccc}
\underline{\pi}_{3} S^{3} \wedge X_{2}^{+} & \stackrel{r \cdot}{\rightarrow} & \underline{\pi}_{3} S^{3} \wedge X_{2}^{+} \\
d_{2 *} \downarrow & & \downarrow d_{2 *} \\
\underline{\pi}_{3} K & \stackrel{ }{\longrightarrow} & \underline{\pi}_{3} K
\end{array}
$$

commutes.

Proof that 5.7.1 implies 5.4.4' and (5.4.4'). We must show that $d_{2}$ may be chosen so that, if $L_{1}=\hat{c}\left(d_{2}\right)$, then $\left[L_{1}, L_{1}\right] \rightarrow \operatorname{Hom}(\underline{M}, \underline{M})=\mathbf{Q}$ is surjective.

Now, Lemma 5.7.1, together with 2.5.2, implies that

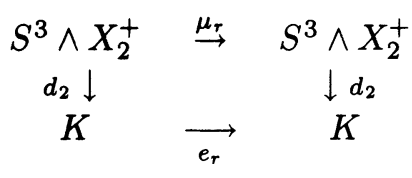


$G$-homotopy-commutes, where $\mu_{r}$ is some $G$-map inducing multiplication by $r$ on $\underline{\pi}_{3} S^{3} \wedge X_{2}^{+}$. (Of course, $\mu_{r}$ is unique, up to $G$-homotopy, but we do not need this.) It follows that $e_{r}$ extends to some $g: L_{1} \rightarrow L_{1}$. Clearly $g_{*}: \underline{H}_{2} L_{1} \rightarrow \underline{H}_{2} L_{1}$ is multiplication by $r$.

Next, we define an equivariant version of Hilton's generalized Hopf invariant. Here, this will be a homomorphism

$$
\mathcal{H}=\mathcal{H}_{Y}: \underline{\pi}_{k} Y \rightarrow \underline{\pi}_{k+1}(Y \times Y, Y \vee Y),
$$

defined for any $k \geq 1$ and any $G$-space $Y$ which is a suspension (of some $G$-space $X)$. Thus, if $p: Y \rightarrow Y \vee Y$ is the pinching map, then $\mathcal{H}_{Y}$ is the composite

$$
\underline{\pi}_{k} Y \stackrel{p *}{\rightarrow} \underline{\pi}_{k}(Y \vee Y) \approx \underline{\pi}_{k} Y \oplus \underline{\pi}_{k} Y \oplus \underline{\pi}_{k+1}(Y \times Y, Y \vee Y) \stackrel{\mathrm{pr}}{\rightarrow} \underline{\pi}_{k+1}(Y \times Y, Y \vee Y)
$$

Our interest in $\not$ derives from the corollary of the next lemma. If $f, g: Y \rightarrow Y$ are $G$-maps, then $f+g$ is defined, as usual, to be the composite

$$
Y \stackrel{p}{\rightarrow} Y \vee Y \stackrel{\{f, g\}}{\rightarrow} Y .
$$

5.7.2. LEMMA. $(f+g)_{*}\left|\operatorname{ker} \not=f_{*}\right| \operatorname{ker} \not H+g_{*} \mid \operatorname{ker} \not$.

5.7.3. COROLlaRY. $\left(f_{r} \wedge \mathrm{id}_{X}\right)_{*} \mid$ ker $\not /$ is multiplication by $r$.

Here, $f_{r}: S^{1} \rightarrow S^{1}$ is as above, and we are identifying $Y=\sum X$ with $S^{1} \wedge X$ as before. Both 5.7.2 and 5.7.3 are standard in the nonequivariant case and are proved in the same way here.

By 5.7.3, Lemma 5.7.1 will follow if we can choose $d_{2}: S^{3} \wedge X_{2}^{+} \rightarrow K$ (lifting $\underline{\partial}_{2}$ and) so that $d_{2 *}\left(\underline{\pi}_{3} S^{3} \wedge X_{2}^{+}\right) \subset \operatorname{ker} \nvdash_{K}$, where $\not_{K}: \underline{\pi}_{3} K \rightarrow \underline{\pi}_{4}(K \times K, K \vee K)$. We next state a computational result which allows us to achieve the desired choice of $d_{2}$.

5.7.4. LEMMA. (a) $\underline{\pi}_{4}(K \times K, K \vee K)=\underline{M} \otimes \underline{M}$.

(b) Set $J=S^{2} \wedge X_{0}^{+}$. Then, $\underline{\pi}_{4}(J \times J, J \vee J)=\underline{F}_{0} \otimes \underline{F}_{0}$.

(c) Let $j: J \rightarrow K$ be the standard inclusion ( $J$ is the base of the mapping cone $K)$, and recall that $\underline{\varepsilon}: \underline{F}_{0} \rightarrow \underline{M}$ comes from the resolution of $\underline{M}$. Then, the following diagram commutes.

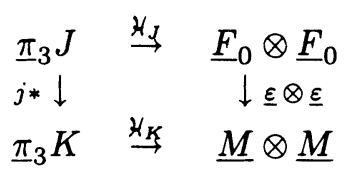

(d) $\nvdash_{K}\left(\underline{\pi}_{3} K\right)=(\underline{\varepsilon} \otimes \underline{\varepsilon}) \nvdash_{J}\left(\underline{\pi}_{3} J\right)$.

We shall leave (a)-(c) as an exercise.

ProOF OF 5.7.4(d). (d) asserts that, for each $H \leq G$,

$$
\text { image } \mathfrak{H}_{K}(G / H)=\operatorname{image}\left((\underline{\varepsilon} \otimes \underline{\varepsilon}) \circ \nvdash_{J}\right)(G / H) \text {. }
$$

By 5.7.4(c), the inclusion $\supseteq$ holds, so we must prove the reverse.

Note that

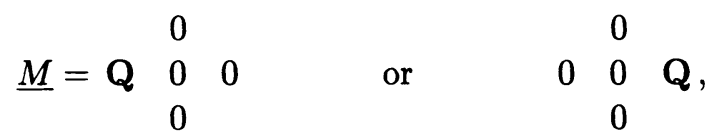


so that in either case $\underline{M} \otimes \underline{M}=\underline{M}$, and it suffices, to show that $(\underline{\varepsilon} \otimes \underline{\varepsilon}) \circ \nvdash_{J}$ is nonzero.

First, consider

$$
\underline{M}=\mathbf{Q} \begin{array}{lll}
0 & \\
0 & 0 . \\
0 &
\end{array}
$$

In this case, consider $H=G$, and note that $\underline{\varepsilon}(G / H): \underline{F}_{0}(G / H) \rightarrow \underline{M}(G / H)$ is the identity map $\mathbf{Q} \rightarrow \mathbf{Q}$. Correspondingly, $J^{G}=S^{2}$, and the map $\left((\underline{\varepsilon} \otimes \underline{\varepsilon}) \circ \mathcal{H}_{J}\right)(G / G)$ is just the standard (rational) Hopf homomorphism $h: \pi_{3} S^{2} \rightarrow \mathbf{Q}$, well known to be nontrivial.

Next, when

$$
\underline{M}=\begin{array}{ll}
0 & 0 \\
& 0 \\
& 0
\end{array}
$$

consider $H=e$. In this case, $\underline{\varepsilon}(G / H)$ is the standard augmentation $\eta: \mathbf{Q}[G] \rightarrow \mathbf{Q}$. Correspondingly, $J^{e}=J=S^{2} \wedge G^{+}$. Let $\alpha$ denote the composite

$$
S^{2}=S^{2} \wedge e \hookrightarrow S^{2} \wedge G^{+}=J
$$

and observe that the diagram

$$
\begin{array}{ccc}
\pi_{3} S^{2} & \stackrel{\alpha_{*}}{\rightarrow} & \pi_{3} J \\
h \downarrow & & \downarrow \nLeftarrow(G / e) \\
\pi_{4}\left(S^{2} \times S^{2}, S^{2} \vee S^{2}\right) & \stackrel{(\alpha \times \alpha)_{*}}{\rightarrow} & \pi_{4}(J \times J, J \vee J)
\end{array}
$$

commutes. It is easy to see that $(\underline{\varepsilon} \otimes \underline{\varepsilon})(G / e)$ takes $\operatorname{im}(\alpha \times \alpha)_{*}$ onto $\mathbf{Q}=\underline{M}(G / e)$, and so it must do the same to im $\not{H}(G / e)$. This completes the proof of 5.7.4(d).

Completing the proof of Lemma 5.7.1. As already observed (following 5.7.3), 5.7.1 will follow if we can choose $d_{2}$ lifting $\underline{\partial}_{2}$ such that $d_{2 *}\left(\underline{\pi}_{3} S^{3} \wedge X_{2}^{+}\right) \subset$ ker $\nLeftarrow$.

Begin by choosing any $\bar{d}_{2}: S^{3} \wedge X_{2}^{+} \rightarrow K$ that lifts $\underline{\partial}_{2}$ (or splits hur)-see 5.4. This gives a diagram

$$
\underline{F}\left[X_{2}\right]=\underline{\pi}_{3} S^{3} \wedge X_{2}^{+} \stackrel{\bar{d}_{2 *}}{\rightarrow} \begin{gathered}
\underline{\pi}_{3} K \\
j_{*} \uparrow
\end{gathered}-\underset{\underline{\pi}_{3} J}{\stackrel{\not H}{\rightarrow}} \rightarrow \underline{M} \otimes \underline{M}
$$

By 5.7.4(d) and the fact that $\underline{F}\left[X_{2}\right]$ is projective, we may complete the solid-arrow diagram with some map $\underline{\delta}$. Choose $d_{2}$ so that

$$
d_{2 *}=\bar{d}_{2 *}-j_{*} \circ \underline{\delta}
$$

which can be done by 2.5.2.

It is easy to see that hur $\circ j_{*}=0$, so that $d_{2}$ splits hur just as $\bar{d}_{2}$ does. Moreover, clearly, im $d_{2 *} \subset$ ker $\nVdash$, as required. Thus, the proofs of 5.7.1, 5.4.4', $\left(5.4 .4^{\prime \prime}\right), 5.2 .1$, and, finally, 5.1 are complete. 


\section{REFERENCES}

[A] James E. Arnold, On Steenrood's problem for cyclic p-groups, Canad. J. Math. 29 (1977), 421-428.

[B] G. Bredon, Equivariant cohomology theories, Lecture Notes in Math., vol. 34, SpringerVerlag, New York, 1967.

[C1] G. Carlsson, A counterexample to a conjecture of Steenrod, Invent. Math. 64 (1981), 171-174.

[C2] __, An Adams-type spectral sequence for change of rings, Houston J. Math. 4 (1978), 541-550.

[Co] G. Gooke, Replacing homotopy actions by topological actions, Trans. Amer. Math. Soc. 237 (1978), 391-406.

[E] A. Elmendorf, Systems of fixed point sets, Trans. Amer. Math. Soc. 277 (1983), 275-284.

[H] P. J. Hilton, Homotopy theory and duality, Gordon \& Breach, New York, 1965.

[K1] P. J. Kahn, Steenrod's problem and k-invariants of certain classifying spaces, Algebraic $K$-theory. Part II, Lecture Notes in Math., vol. 967, Springer-Verlag, New York, 1982.

[K2] _ Equivariant homology decompositions, Trans. Amer. Math. Soc. 298 (1986), 273287.

[L] W. Lück, Diplomarbeit, Univ. of Göttingen, 1981.

[M] S. Mac Lane, Homology, Academic Press and Springer-Verlag, New York, 1963.

$[\mathbf{R} \& \mathbf{T}]$ M. Rothenberg and G. Triantafillou, An algebraic model for simple homotopy types, Univ. of Minnesota Math. Report 83-112, 1984.

[S] J. Smith, Equivariant Moore spaces, 1982 (preprint).

[Sw] R. Swan, Invariant rational functions and a problem of Steenrod, Invent. Math. 7 (1969), $148-158$.

[T1] G. Triantafillou, Equivariant minimal models, Trans. Amer. Math. Soc. 274 (1982), 509532.

[T2] _ G-spaces with prescribed equivariant cohomology, Univ. of Minnesota (preprint).

[T3] _ Rationalization of Hopf G-spaces, Math. Z. 182 (1983), 485-500.

[V] P. Vogel, On Steenrod's problem for non-abelian finite groups, Algebraic Topology, Aarhus 1982, Lecture Notes in Math., vol. 1051, Springer-Verlag, New York, 1984.

[W] C. T. C. Wall, Finiteness conditions for CW complexes. II, Proc. Roy. Soc. A 295 (1966), 129-139.

[Wn] S. Waner, Equivariant homotopy theory and Milnor's theorem, Trans. Amer. Math. Soc. 258 (1980), 351-368. 